\title{
Breakup, Coalescence, and Migration Regularity of Bubbles under Gas-Liquid Swirling Flow in Gas-Liquid Cylindrical Cyclone
}

\author{
Lele Yang, Li Zou,* Yong Ma, Jing Wang, and Jingyu Xu
}

Cite This: Ind. Eng. Chem. Res. 2020, 59, 2068-2082

Read Online

ABSTRACT: This study investigates the bubble behavior in the lower part of the gasliquid cylindrical cyclone experimentally and numerically. Malvern RTsizer and electrical resistance tomography were used to determine bubble size distribution and void fraction distribution, respectively. A discrete phase model was used to simulate the swirling flow field characteristics. The results indicated that as the gas flow rate increased, the small and medium-sized bubbles initially broke up and subsequently coalesced, whereas as the liquid flow rate increased, they only tended to break up. The gas core narrowed as the gas and liquid flow rates increased. The optimal gas-liquid separation performance was achieved when the liquid level was near $0.82 \mathrm{~m}$. The separation mechanism of bubbles was analyzed via experimental observations and the simulated flow field distribution. An effective mechanism model based on bubble migration analysis was developed to predict the critical diameter of bubbles that can be separated.

\section{INTRODUCTION}

Cyclone separators were introduced for the dedusting of gas in 1885. Since then, they have been commonly used in the petroleum, chemical, mineral, and environmental industries. Numerous experimental and theoretical studies on cyclone separation have been conducted, and many separation theories have been proposed. Most of these theories focus on gas-solid and liquid-solid separation. Relatively mature design methods have been developed and are being applied successfully in these areas. However, the complex multiphase hydrodynamics and variability of the operating conditions make performance prediction of the gas-liquid cyclone very challenging. ${ }^{1,2}$ Thus, the oil industry still relies on conventional vessel-type separators to separate natural gas from the gas-liquid mixtures delivered from oil/gas wells. ${ }^{3}$ To realize highly efficient gasliquid separation, researchers must further study the gas-liquid cyclone separation mechanism.

There are several types of gas-liquid cyclones, as shown in Figure 1. The spiral vane-type gas-liquid cyclone illustrated in Figure $1 \mathrm{a}$ is suitable for downhole separation. This configuration has a low swirl intensity; thus, its treatment capacity is low. Figure $1 \mathrm{~b}$ shows the swirl vane-type gas-liquid cyclone, which includes a circle of blades with a certain internal arrangement. It is widely used in wet desulphurization. Another type that was recently proposed is the guide vanetype gas-liquid cyclone, which is shown in Figure 1c. It is more effective under space-limited conditions but cannot easily obtain pure gas. The most widely used type is the gas-liquid cylindrical cyclone (GLCC), ${ }^{4}$ which is derived from a hydrocyclone with cylindrical and conical sections. As shown in Figure 1d, the GLCC comprises a vertical pipe with a downward inclined tangential inlet located approximately at the mid-height, as well as two outlets: one located at the top and the other at the bottom of the pipe. The tangential inlet provides swirling motion and thus produces centrifugal forces for the separation of the gas-liquid mixture. During regular operation, the liquid exits from the bottom, while the gas escapes from the top. However, under certain operating conditions, some liquid may be entrained with the gas flow, causing liquid carry-over (LCO). In this situation, some gas may escape with the liquid, causing gas carry-under (GCU). In the upper part of the GLCC, droplets are pushed toward the wall to form the upper swirling liquid film (USLF). In the lower part of the GLCC, the flow comprises a liquid phase, through which bubbles migrate radially toward the pipe axis, forming a gas core.

For GLCCs, many experimental and numerical studies have been conducted regarding the relevant parameters of design and operation. The geometric parameters can affect the phase split in the GLCC, such as the diameter and length of the GLCC body, inclination angle, number of inlets, inlet nozzle geometry, and inlet location. Previous results indicate that a converging inlet nozzle and multiple inlets can be used to improve the separation efficiency of the GLCC. ${ }^{5}$ Kouba et al. ${ }^{6}$ observed that the optimal inlet angle is $27^{\circ}$, which can retard significantly the onset of LCO. However, increasing the inlet

Received: September 25, 2019

Revised: November 27, 2019

Accepted: January 14, 2020

Published: January 14, 2020 


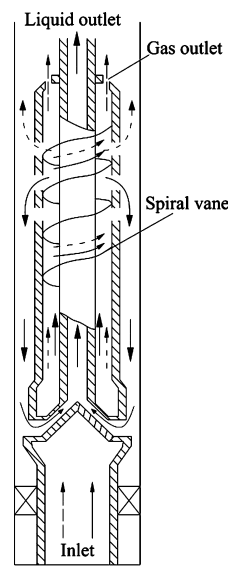

(a)

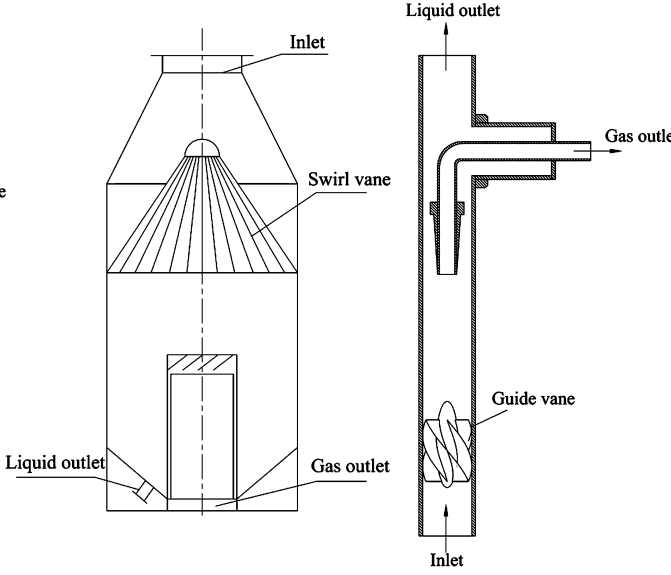

(b)

(c)

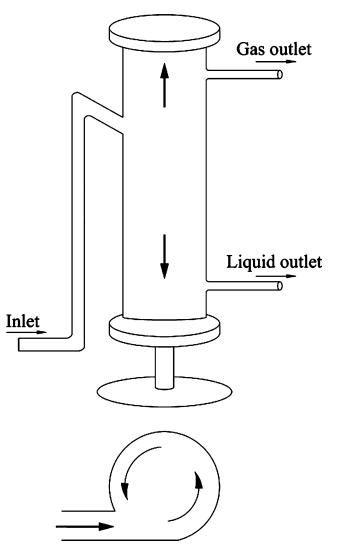

(d)

Figure 1. Different types of gas-liquid cyclones: (a) spiral vane, (b) swirl vane, (c) guide vane, (d) cylindrical.

angle excessively results in the GCU phenomenon. ${ }^{7}$ By adopting computational fluid dynamics modeling, Carlos Berrio et al. ${ }^{8}$ obtained the optimum configuration corresponding to an angle of $27^{\circ}$ with an inlet location $10 \mathrm{~cm}$ above the center, dual inlet with $20 \mathrm{~cm}$ spacing between the two legs, 3.5 $\mathrm{cm}$ diameter nozzle, and $15 \%$ pipe area volute inlet. Moreover, the operational parameters, including the gas and liquid flow rates, ${ }^{9}$ the operating pressure, ${ }^{10}$ and fluid properties, ${ }^{11,12}$ are directly related to the separation efficiency of the GLCC.

LCO and GCU are two phenomena that limit the separation performance of a GLCC. Kolla et al. ${ }^{13}$ observed that the liquid carried with the gas stream existed in the form of a liquid film or droplets. Wang et al. ${ }^{14}$ evaluated the improvement of the GLCC performance in terms of an operational envelope for LCO and quantified the liquid extraction from the gas stream. According to the experimental observations, Yue et al. ${ }^{15}$ defined four flow regimes of the USLF, including the swirling flow, churn flow, annular flow, and ribbon flow. They used the Eulerian wall film model to examine the effects of the fluid properties and operational conditions on the flow behavior of the USLF. According to an analysis of the droplet trajectory, Chirinos et al. ${ }^{16}$ and Molina et al. ${ }^{9}$ developed mechanistic models for predicting the amount of LCO. Regarding the GCU, a few studies were performed to predict the gas-liquid interface using CFX software. ${ }^{17,18}$

Most of the previous research has been limited to the effects of the geometric and operational parameters on the separation performance of GLCCs. The relationship between the swirling flow field and the separation performance has seldom been studied. Moreover, most research has been conducted to elucidate the droplet migration regularity, flow behavior of the liquid film, and parameters influencing the LCO in the upper part of the GLCC. Insufficient attention has been paid to the separation characteristics of bubbles in the lower part of the GLCC. In the present study, the bubble behavior in the lower part of the GLCC (particularly the breakup, coalescence, and migration of bubbles) was investigated via experiments and numerical simulations. In the experiments, the bubble size distribution and void fraction distribution were measured via a Malvern RTsizer and electrical resistance tomography (ERT), respectively. The discrete phase model (DPM) coupled with the Reynolds stress model (RSM) was used to simulate the swirling flow field characteristics. Furthermore, a mechanism model was developed for predicting the critical diameter of bubbles that can be separated, according to an analysis of the bubble migration. The results and conclusions of this study may provide guidance for the design and operation of GLCCs to minimize the GCU.

\section{EXPERIMENTAL INVESTIGATION}

2.1. Measuring Technique. Numerous techniques have been applied for measuring the particle size distribution. Among them, photographic methods and the laser diffraction technique can achieve fast online measurements. Photographic methods are more suitable for measuring large particles, as small particles may not be detected if they are outside the field of the camera lens. For the laser diffraction technique, the concentration of the measured particles should be low. This is because the algorithms of this technique assume that the laser light is scattered only by one particle. Multiple scattering of laser light by many particles may occur at high concentrations. ${ }^{19}$ Simmons et al. ${ }^{20}$ compared the measuring methods of Malvern and the laser backscatter technique and found that the Malvern method performed well when the volume fraction was less than $3 \%$. Liu et al. ${ }^{21}$ used a Malvern RTsizer to measure the bubble size distribution and investigated the breakup and coalescence regularity of bubbles in a vane-type swirling flow field. In this study, the bubble concentrations were relatively low, and the bubble size could be small. Thus, the bubble size distributions were measured using a Malvern RTsizer via the laser diffraction technique.

The measurement principle of a Malvern RTsizer is shown in Figure 2. Particles flow through the beam scatter light

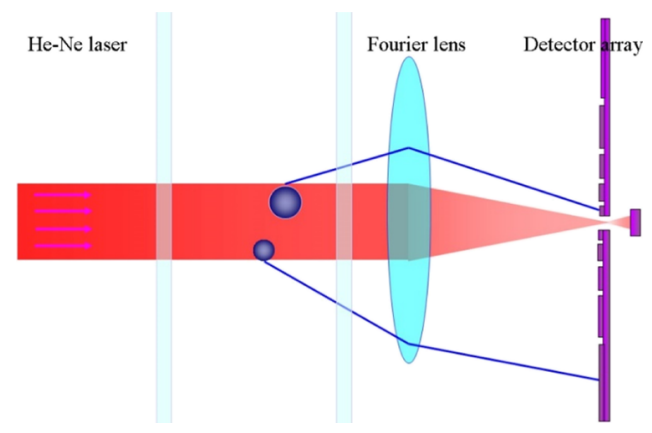

Figure 2. Measurement principle diagram of a Malvern RTsizer. 
released by a $\mathrm{He}-\mathrm{Ne}$ laser. The light-scattering phenomenon occurs owing to diffraction. The particle size is inversely proportional to the diffraction angle. The scattered light is focused by a Fourier lens and then detected by an annular detector array. After the scattered light is converted into a farfield diffraction pattern, the particle size distribution can be determined according to the energy spectrum. The particle size distribution parameters $d_{\min }, d_{32}, d_{50}$, and $d_{\max }$ can be calculated. Here, $d_{32}$ represents the Sauter mean diameter, and 10,50 , and $95 \%$ (volume fraction) of the particles have diameters less than $d_{\min }, d_{50}$, and $d_{\max }$, respectively.

ERT is an imaging technique used for multiphase flow measurement and visualization. It can provide cross-sectional images of dispersed phases in terms of distribution and concentration through measurement of the voltage at the electrodes. $^{22,23} \mathrm{Jia}$ et al. ${ }^{24}$ found that ERT could measure a full void-fraction range of the dispersed phase from 0 to 1 when the conductive liquid remained in a continuous phase in the mixture. In this study, ERT was employed to measure the phase distribution in the lower part of the GLCC. Dual-plane ERT sensors were placed $0.05 \mathrm{~m}$ apart. Each plane contained 16 titanium-alloy rectangular electrodes for obtaining the conductivity distribution. According to the Maxwell relationship, the gas volume fraction can be calculated using the measured conductivity distribution. A slight reference measurement error can lead to significant conductivity and thus to an inaccurate gas volume fraction. ${ }^{25}$ Thus, background measurements with pure water were performed before each test. The reference measurement error did not exceed $1 \%$.

2.2. Experimental Setup. A schematic of the experimental setup is shown in Figure 3. The internal diameter of the GLCC body was $0.05 \mathrm{~m}$, and its total height was $1.98 \mathrm{~m}$. The inlet pipe had a $0.025 \mathrm{~m}$ inner diameter. Three types of inlet nozzles were employed in this study. A nozzle without a reduced diameter was denoted as nozzle 1 . Nozzles with a volute inlet

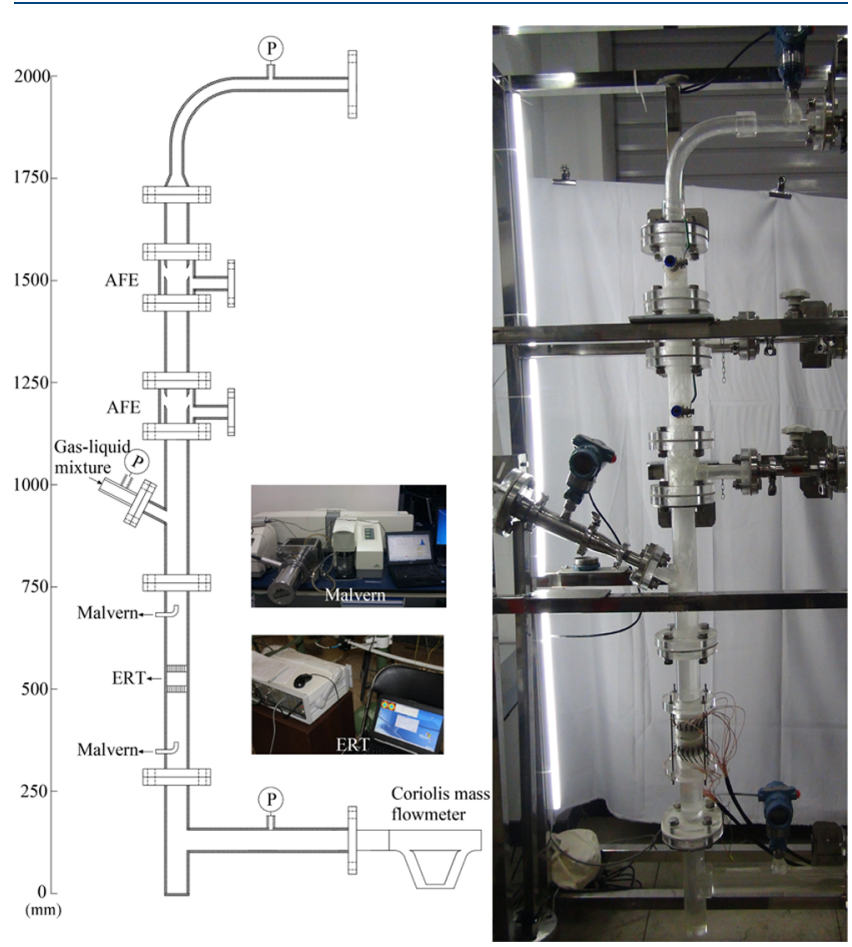

Figure 3. Schematic and photographs of the experimental setup. of 68 and $37 \%$ of the pipe area were denoted as nozzle 2 and nozzle 3, respectively.

For this study, air and tap water were selected as the test fluids. The density of the tap water was $999.0 \mathrm{~kg} / \mathrm{m}^{3}$, with a viscosity of $1.0 \mathrm{mPa} \cdot \mathrm{s}$. The density of the air was $1.205 \mathrm{~kg} / \mathrm{m}^{3}$, with a viscosity of $1.81 \times 10^{-3} \mathrm{mPa} \cdot \mathrm{s}$. The air-water surfacetension coefficient was $0.072 \mathrm{~N} / \mathrm{m}$. The gas flow rates varied from 41 to $112 \mathrm{~m}^{3} / \mathrm{h}$, and the liquid flow rates varied from 0.34 to $0.98 \mathrm{~m}^{3} / \mathrm{h}$. In the experiments, the GLCC operated almost at atmospheric pressure. All of the experiments were performed at a temperature of $20{ }^{\circ} \mathrm{C}$. The test conditions in the experiments are summarized in Table 1.

Compressed air supplied by an air compressor was mixed with the tap water pumped from a water tank, and the mixture was passed through an atomizing nozzle before entering the GLCC. The inlet air after filtration was measured by a thermal gas mass flowmeter, while the inlet water was metered by a Coriolis mass flowmeter. The flow rate and density of the mixture flowing through the liquid outlet were determined using a Coriolis mass flowmeter. Three pressure gauges were placed at the inlet and each outlet to monitor the pressure drop in the GLCC. Two annular film extractors (AFEs) were used to remove the liquid film. A volumetric measurement technique was employed to measure the flow rates of the liquid flowing out through the gas outlet and each AFE. Details regarding the sampling method for the Malvern RTsizer were presented in previous reports, ${ }^{21,26}$ and the method has proven to be reliable. Two pieces of sampling equipment placed 0.37 and $0.76 \mathrm{~m}$ above the bottom of the GLCC were connected to the Malvern RTsizer. As shown in Figure 4, three sampling locations in the section were selected to detect changes in the bubble size distribution along the radius. The diameter of the sampling pipe was $8 \mathrm{~mm}$. Additionally, the sampling pipe was short enough and its outlet was close enough to the measuring zone to reduce potential changes of the bubble distribution induced by physical factors. Dual-plane ERT sensors were placed 0.50 and $0.55 \mathrm{~m}$ above the bottom of the GLCC.

In the measurement process, the inlet and outlet flow rates and pressures were recorded over $40 \mathrm{~s}$ by a data-acquisition card. The average of the $40 \mathrm{~s}$ datasets was calculated to obtain the final experimental data. The bubble size distribution was measured several times for each case. A total of 8000 dualframe voltage measurements were conducted to obtain an accurate phase distribution for each flow condition.

\section{NUMERICAL SIMULATIONS}

3.1. Discrete Phase Model. In this study, numerical simulations were conducted using ANSYS Fluent 14.5. The DPM in fluent was established according to the EulerLagrange method. Taking a spatial point as an object, the continuous phase was described from the Euler viewpoint. Taking a single particle as an object, the discrete phase was described from the Lagrange viewpoint.

The continuity equation and momentum equation of the continuous phase are, respectively, expressed as

$$
\begin{aligned}
& \frac{\partial \bar{u}_{i}}{\partial x_{i}}=0 \\
& \frac{\partial \bar{u}_{i}}{\partial t}+\overline{u_{j}} \frac{\partial \bar{u}_{i}}{\partial x_{j}}=-\frac{1}{\rho} \frac{\partial \bar{p}}{\partial x_{i}}+\nu \frac{\partial^{2} \overline{u_{i}}}{\partial x_{j} \partial x_{j}}-\frac{\partial}{\partial x_{j}} \overline{u_{i}^{\prime} u_{j}^{\prime}}
\end{aligned}
$$


Table 1. Test Conditions in the Experiments

$\begin{array}{ccccc}\text { inlet gas flow rate }\left(\mathrm{m}^{3} / \mathrm{h}\right) & \text { inlet liquid flow rate }\left(\mathrm{m}^{3} / \mathrm{h}\right) & \text { liquid level }(\mathrm{m}) & \text { temperature }\left({ }^{\circ} \mathrm{C}\right) & \text { inlet nozzle } \\ 41-112 & 0.34-0.98 & 0.31-0.94 & 20 & \text { nozzle } 1,2,3\end{array}$

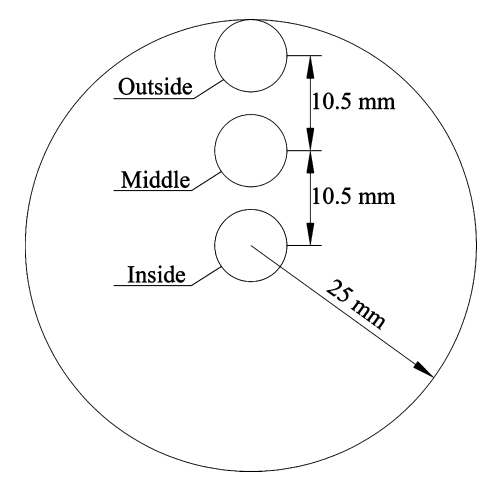

Figure 4. Sampling locations for the Malvern RTsizer.

where $\overline{u_{i}}$ represents the mean velocity; $\rho$ represents the continuous-phase density; $\nu$ represents the continuous-phase kinematic viscosity; $\bar{p}$ represents the mean pressure; and $\overline{u_{i}^{\prime} u_{j}^{\prime}}$ represents Reynolds stress tensor.

The trajectory of dispersed particles can be solved using the force balance equation of the particle and can be expressed in the following form (for the $x$-direction in a Cartesian coordinate system)

$$
\frac{\mathrm{d} u_{\mathrm{p}}}{\mathrm{d} t}=f_{\mathrm{D}}\left(u-u_{\mathrm{p}}\right)+\frac{\left(\rho_{\mathrm{p}}-\rho\right) g_{x}}{\rho_{\mathrm{p}}}+f_{x}
$$

where $u$ represents the continuous-phase velocity; $u_{\mathrm{p}}$ represents the particle velocity; $\rho_{\mathrm{p}}$ represents the particle density; $f_{\mathrm{x}}$ represents the force per unit particle mass, which includes the lift force, Magnus force, and Saffman force; and $f_{\mathrm{D}}$ is the drag coefficient, which is defined as

$$
\begin{aligned}
& f_{\mathrm{D}}=\frac{18 \mu}{\rho_{\mathrm{p}} d_{\mathrm{p}}^{2}} \frac{C_{\mathrm{D}} R e}{24} \\
& R e=\frac{\rho d_{\mathrm{p}}\left|\vec{u}_{\mathrm{p}}-\vec{u}\right|}{\mu}
\end{aligned}
$$

here, $C_{\mathrm{D}}$ is the drag coefficient correlated with $R e$, which is calculated as

$$
C_{\mathrm{D}}=n_{1}+\frac{n_{2}}{R e}+n_{3}
$$

where $n_{1}, n_{2}$, and $n_{3}$ are the constants given by Morsi and Alexander. ${ }^{27}$

The Taylor analogy breakup (TAB) model was used to simulate the breakup of dispersed particles. The basic principle of the TAB model is to calculate the oscillation and deformation of particles. When the oscillation reaches the critical value, particles break up. The forced damped vibration governing equation for the particle is given as follows

$$
F-k x-d_{\mathrm{p}} \frac{\mathrm{d} x}{\mathrm{~d} t}=m \frac{\mathrm{d}^{2} x}{\mathrm{~d} t^{2}}
$$

where $m$ represents the mass of the particle, $F$ represents the external force acting on the particle, $x$ represents the oscillating deformation, and $d_{\mathrm{p}}$ represents the diameter of the particle. When $x>0.25 d_{\mathrm{p}}$, the particle is assumed to have broken up.

The coalescence of the particles is described by the model proposed by O'Rourke. ${ }^{28}$ The model proposes the concept of parcels, that is, groups of particles, to reduce the amount of calculation. Rather than judging whether the orbits of two parcels overlap, O'Rourke adopted the stochastic method to calculate the probability of collisions. He also suggested that two parcels might collide only if they exist in the same grid. The collision frequency is calculated as follows

$$
P_{1}=\frac{\pi\left(r_{1}+r_{2}\right)^{2} v_{\text {rel }} \Delta t}{V}
$$

where $r_{1}$ and $r_{2}$ represent the radii of the two parcels, $V$ represents the volume of the grid, and $v_{\text {rel }}$ represents the relative velocity between the two parcels.

The collision parameter $b$ is defined as follows

$$
b=\left(r_{1}+r_{2}\right) \sqrt{Y}
$$

where $Y$ represents the mean variation.

The critical collision parameters are as follows

$$
b_{\text {crit }}=\left(r_{1}+r_{2}\right) \sqrt{\min \left(1.0, \frac{2.4 f}{W e}\right)}
$$

where $f$ is a function of $r_{1} / r_{2}$ and $W e$ represents the collision Weber number.

If $b>b_{\text {crit }}$ the two parcels collide frontally and rebound. If $b$ $<b_{\text {crit }}$ the collision results in the coalescence of the two parcels.

According to the conservation of momentum and kinetic energy, the parcel velocity after the collision can be calculated as follows

$$
v_{1}^{\prime}=\frac{m_{1} v_{1}+m_{2} v_{2}}{m_{1}+m_{2}}+\frac{m_{2}\left(v_{1}-v_{2}\right)}{m_{1}+m_{2}}\left(\frac{b-b_{\text {crit }}}{r_{1}+r_{2}-b_{\text {crit }}}\right)
$$

where $m_{1}$ and $m_{2}$ represent the masses of the two parcels and $v_{1}$ and $v_{2}$ represent the velocities of the two parcels before collision.

3.2. Computational Fluid Dynamics Grids and Simulation Method. Details regarding the structure are presented in Figure 3. The structure is complex at the inlet and AFEs. Therefore, a compact tetrahedral grid was generated in these parts, while the remaining parts were partitioned using a structured grid, as shown in Figure 5. After verifying the grid independence of the simulations, a hybrid grid with $1,557,350$ cells was selected as the final meshing scheme. For the selected grid, the minimum orthogonal quality was 0.23 , and the maximum skewness was 0.46 . The worst size change was 1.57 , and the worst taper was 0.26 .

A velocity inlet boundary condition was applied at the inlet. The pressure outlet boundary condition was set according to the measurements under experimental conditions. For the dispersed phase, particle injection was employed, and the inlet particle size was determined according to experimental results measured by the Malvern RTsizer. The standard wall function was imposed to simulate the flow in the near-wall region. The 


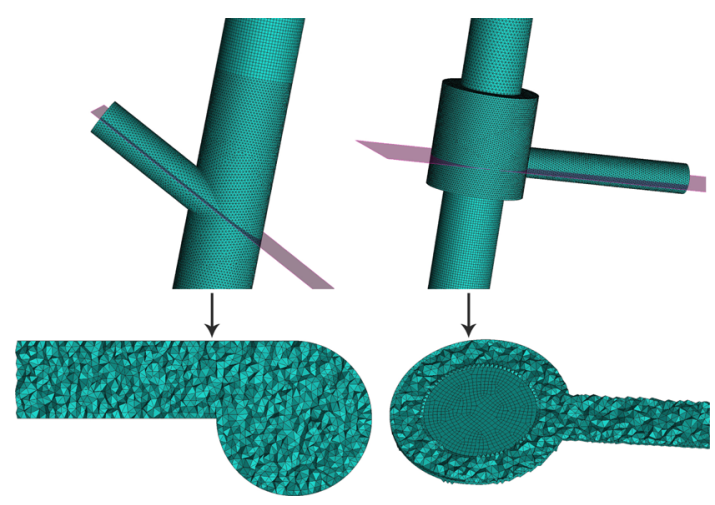

Figure 5. Grid of the GLCC.

hydraulic diameter was set as $0.025 \mathrm{~m}$, which was equal to the inner diameter of the inlet pipe. Considering the unsteadiness of the flow in the GLCC, the numerical simulations were conducted in the unsteady mode, with a timestep of $0.5 \mathrm{~ms}$.

3.3. Turbulent Model. The RNG $k-\varepsilon$ and RSM models are the most common turbulence models used in the numerical study of swirling flow. The RNG $k-\varepsilon$ model includes a mainstream time-averaged strain rate and considers turbulent vortices, yielding better results in flow simulation with severe bending streamlines and a high strain rate. The RSM model can simulate the characteristics of anisotropic turbulence. To select an applicable turbulence model, a comparison between the RNG $k-\varepsilon$ and RSM model results was performed. As shown in Figure 6, the pressure drops between the inlet and

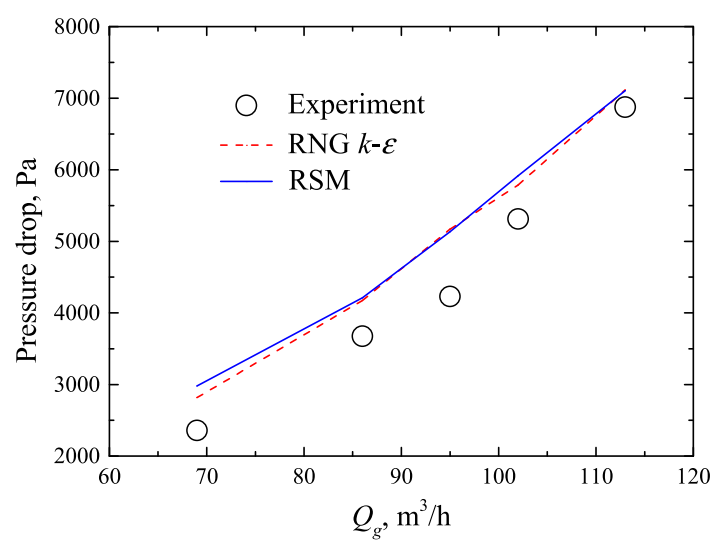

Figure 6. Comparison between the pressure drops for the RNG $k-\varepsilon$ and RSM turbulence models.

liquid outlet were nearly identical for the two turbulence models, and both were slightly less than the experimental data. This is because the transport phenomena in the GLCC are very complex. Compared with the swirl-free axial pipe flows, the swirl presence increases significantly turbulence levels. ${ }^{1}$ The turbulence models cannot totally reproduce the swirling hydrodynamics.

For the two turbulence models, the liquid concentrations in three different sections $0.40,0.60$, and $0.80 \mathrm{~m}$ above the bottom of the GLCC are depicted in Figure 7. The RNG $k-\varepsilon$ model failed to obtain the proper gas phase distribution, and the RSM model predicted a gas core similar to the experimental observation. For the GLCC presented in this work, the swirling flow field was strong and the turbulence was highly anisotropic. Escue and $\mathrm{Cui}^{29}$ recognized that the RSM

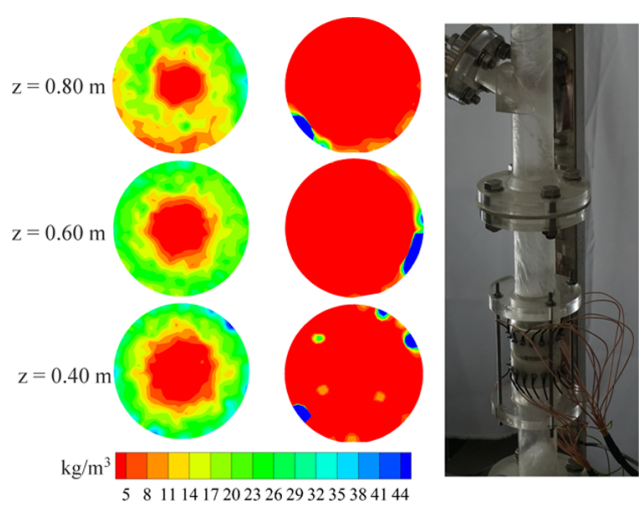

Figure 7. Comparison between the liquid volume concentrations for the RNG $k-\varepsilon$ and RSM turbulence models.

model was more suitable for a strong swirling flow field than the RNG $k-\varepsilon$ model. Besides, the RSM model has better performance generally due to its anisotropic hypothesis and accounts for the effects of streamline curvature, swirling, rotation, and rapid changes in strain rate. Therefore, the RSM model is a better choice for simulating the swirling flow in the GLCC. The transport equations of the RSM model are as follows

$$
\begin{aligned}
& \frac{\partial}{\partial t}\left(\rho \overline{u_{i}^{\prime} u_{j}^{\prime}}\right)+\frac{\partial}{\partial x_{k}}\left(\rho u_{k}^{\prime} \overline{u_{i}^{\prime} u_{j}^{\prime}}\right)=P_{i j}+D_{\mathrm{T} i j}+\phi_{i j}-\varepsilon_{i j}+F_{i j} \\
& P_{i j}=-\rho\left(\overline{u_{i}^{\prime} u_{k}^{\prime}} \frac{\partial u_{j}}{\partial x_{k}}+\overline{u_{j}^{\prime} u_{k}^{\prime}} \frac{\partial u_{i}}{\partial x_{k}}\right) \\
& D_{\mathrm{T} i j}=-\frac{\partial}{\partial x_{k}}\left[\rho \overline{u_{i}^{\prime} u_{j}^{\prime} u_{k}^{\prime}}+\overline{p\left(\delta_{k j} u_{i}^{\prime}+\delta_{i k} u_{j}^{\prime}\right)}\right] \\
& \phi_{i j}=p\left(\frac{\partial u_{i}^{\prime}}{\partial x_{j}}+\frac{\partial u_{j}^{\prime}}{\partial x_{i}}\right) \\
& \varepsilon_{i j}=-2 \mu \frac{\partial u_{i}^{\prime}}{\partial x_{k}} \frac{\partial u_{j}^{\prime}}{\partial x_{k}} \\
& F_{i j}=-2 \rho \Omega_{k}\left(\overline{u_{j}^{\prime} u_{m}^{\prime}} \varepsilon_{i k m}+\overline{u_{i}^{\prime} u_{m}^{\prime}} \varepsilon_{j k m}\right)
\end{aligned}
$$

where $P_{i j}$ is the stress generation term, $D_{\mathrm{T} i j}$ is the turbulent diffusion term, $\phi_{i j}$ is the pressure-strain term, $\varepsilon_{i j}$ is the viscous dissipation term, and $F_{i j}$ is the system rotation generation term.

\section{RESULTS AND DISCUSSION}

4.1. Bubble Size Distribution. 4.1.1. Flow Behavior of Bubbles. Figure 8 illustrates the flow behavior of bubbles in the lower part of the GLCC. In these cases, the liquid level was set as $0.76 \mathrm{~m}$, and the gas and liquid flow rates were 69 and 0.98 $\mathrm{m}^{3} / \mathrm{h}$, respectively. The higher Malvern sampling location was set below the free interface $(0.76 \mathrm{~m}$ above the bottom of the GLCC). As shown, the bubbles at the higher Malvern sampling location were generally small. At this location, the bubble size distributions at different positions of the section were similar. Subsequently, bubbles coalesced in the gas-liquid swirling flow; thus, the bubble size distributions became wider. This phenomenon is consistent with the experimental observations. A large number of dispersed bubbles were generated near the free interface. Larger bubbles were pushed to the free interface 

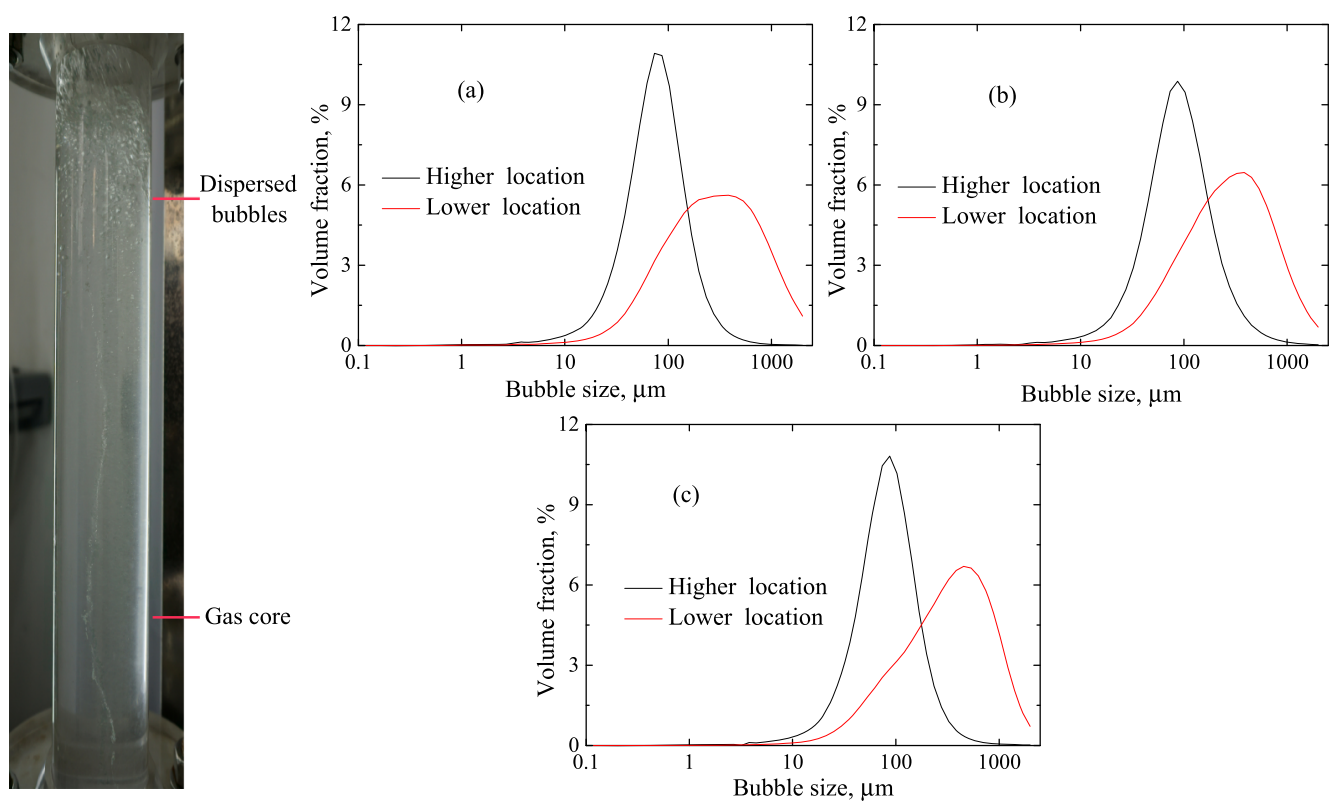

Figure 8. Measured bubble size distributions at different positions of the pipe: (a) outside, (b) middle, (c) inside.

via buoyancy. The residual bubbles measured at the higher Malvern sampling location were relatively small. The bubble density decreased with the increasing distance from the interface. Owing to the centrifugal effect, these bubbles were likely to coalesce in an ordered rotational flow, which increased the size of the bubbles at the lower Malvern sampling location. For additional details regarding the bubble coalescence, please see video "bubble coalescence".

The bubble size distributions at the lower Malvern sampling location were carefully recorded, as shown in Figure 9. Clearly,

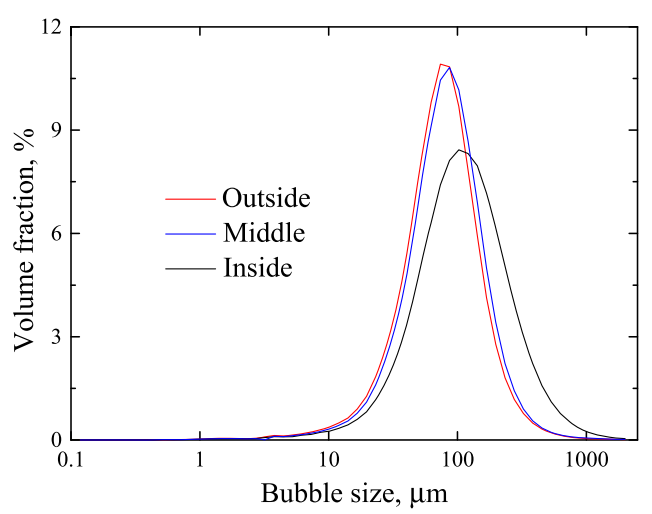

Figure 9. Measured bubble size distributions at the lower Malvern sampling location.

the bubble size distribution inside the pipe was larger than that outside the pipe and in the middle of the pipe. The bubble size distribution outside the pipe was slightly smaller than that in the middle of the pipe. Thus, the centrifugal effect caused by the rotating liquid was more pronounced for the larger bubbles. Thus, the larger bubbles rapidly migrated to the pipe center and accumulated inside the pipe. However, the swirl intensity was insufficient to concentrate small bubbles at the pipe center over a short distance. Thus, the smaller bubbles were dispersed outside the pipe and in the middle of the pipe.

4.1.2. Effects of Gas and Liquid Flow Rates on Bubble Breakup and Coalescence. Figure 10 presents the effect of the gas flow rate on the character diameters at the lower Malvern sampling location. With an increase in the gas flow rate, $d_{\text {min }}$ $d_{32}$, and $d_{50}$ exhibited the same trend: they first decreased and then increased. This phenomenon is theoretically reasonable. When the gas flow rates increased, the shear action on the liquid interface from the gas flow intensified. Bubbles produced near the free interface tended to be smaller and denser, as shown in Figure 11. Additionally, referring to Zhang and $\mathrm{Xu}^{30}$ the reduction of $d_{32}$ indicates the augmentation of the turbulent dissipation energy. Greater turbulent dissipation energy corresponds to a more disordered flow. As indicated by the change of $d_{32}$, the turbulent dispersion of the swirling flow increased as the gas flow rate initially increased. The probability of collision and coalescence between small and medium-sized bubbles decreased correspondingly. These small and medium-sized bubbles were more likely to break up in disordered flow. Thus, $d_{\min }$ and $d_{50}$ first decreased with an increase in the gas flow rate. However, as the gas flow rate increased further, $d_{\min }$ and $d_{50}$ began to increase because the dispersed bubble flow became a relatively ordered swirling flow, as predicted by the augmentation of $d_{32}$. The probability of collision and coalescence between small and medium-sized bubbles began to increase. In summary, small and mediumsized bubbles initially tended to break up and then coalesce as the gas flow rate increased. In contrast to the other character diameters, the maximum bubble diameter $d_{\max }$ decreased gradually with an increase in the gas flow rate. As the gas flow rate increased, the sectional area occupied by the liquid phase decreased; thus, the liquid velocity increased. Additionally, a high gas flow rate promoted an increase in the liquid rotational speed under the effect of the drag force. The augmentation of the swirl intensity caused the bubbles that could be separated to become smaller. The maximum diameter of the bubbles dispersed outside the gas core decreased.

Figure 12 depicts the effect of the liquid flow rate on the character diameters. In contrast to the influence rule of the gas flow rate, all of the character diameters exhibited the following trend: they decreased as the liquid flow rate increased. The reduction of $d_{32}$ indicates that the swirling flow became 

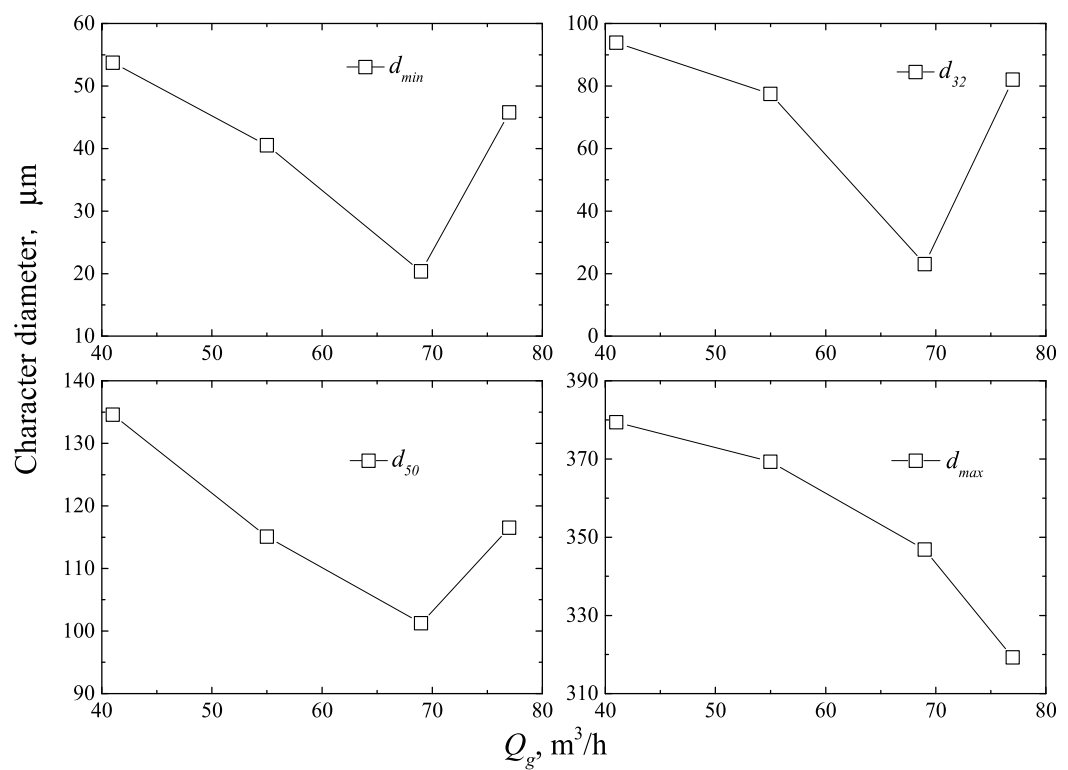

Figure 10. Effect of the gas flow rate on the character diameter at an inlet liquid flow rate of $0.68 \mathrm{~m}^{3} / \mathrm{h}$.

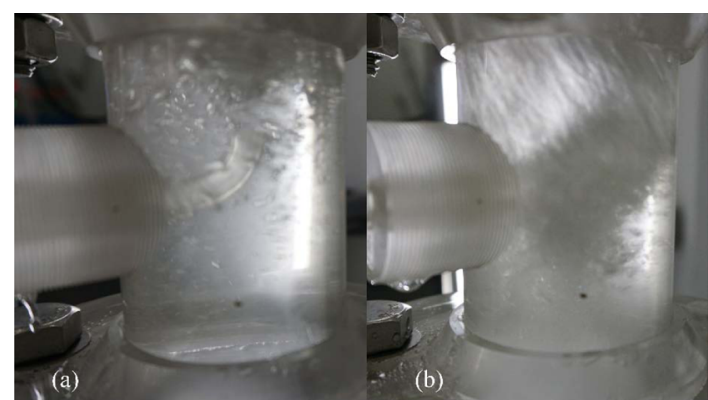

Figure 11. Bubbles near the free interface at different gas flow rates: (a) low, (b) high.

unsteady. It was more difficult for small and medium-sized bubbles to collide and coalesce. Furthermore, the swirl intensity increased with the liquid flow rate. Under a high swirl intensity, most of the bubbles, particularly the large bubbles, were concentrated in the gas core, and the bubble dispersion decreased. Higher liquid flow rates produced a higher phase slip velocity. Then, the shear damage caused by the continuous liquid phase increased, and large bubbles were easily broken up. Therefore, $d_{\min }, d_{50}$, and $d_{\max }$ decreased as the liquid flow rate increased.

4.1.3. Effect of Inlet Nozzle Design on Maximum Bubble Diameter. The inlet nozzle design is crucial for GLCC function because it has a direct correlation with the swirl intensity. To investigate the effect of the inlet nozzle on the bubble behavior, all cases were repeated using three different nozzles. The effect of the inlet nozzle design on the maximum bubble diameter at the lower Malvern sampling location was investigated, and the results are shown in Figure 13. When the inlet gas and liquid flow rates were fixed, the maximum bubble diameter decreased with an increase in the degree of restriction

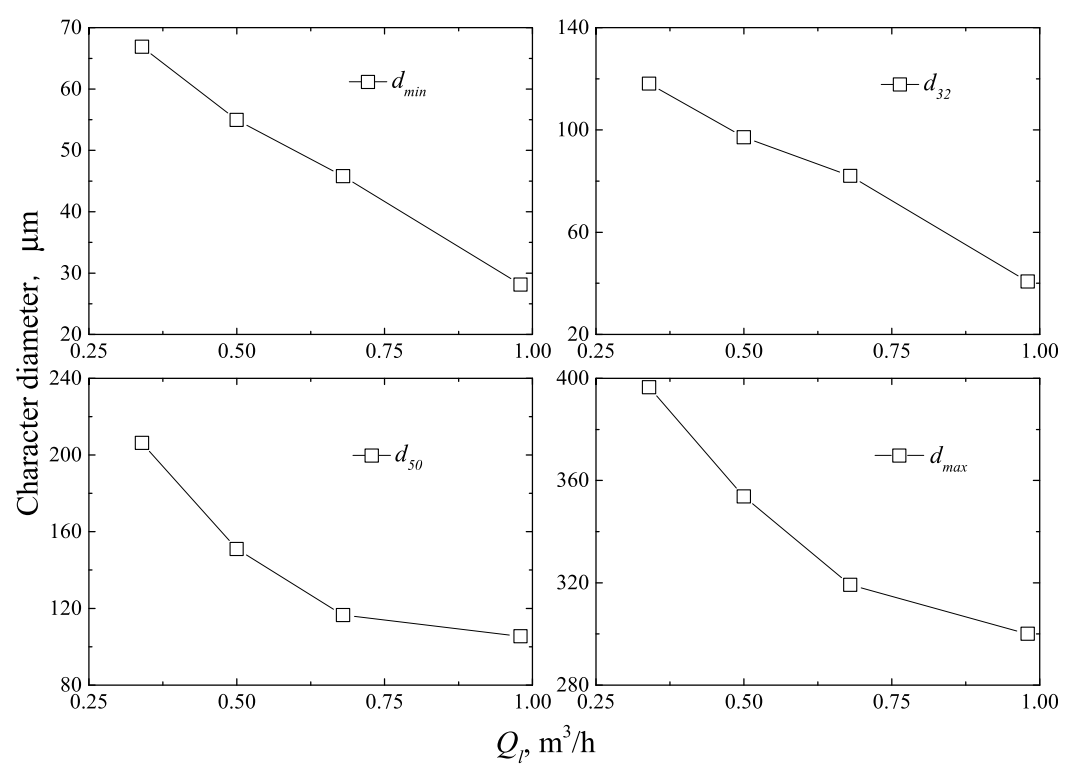

Figure 12. Effect of the liquid flow rate on the character diameter at an inlet gas flow rate of $77 \mathrm{~m}^{3} / \mathrm{h}$. 


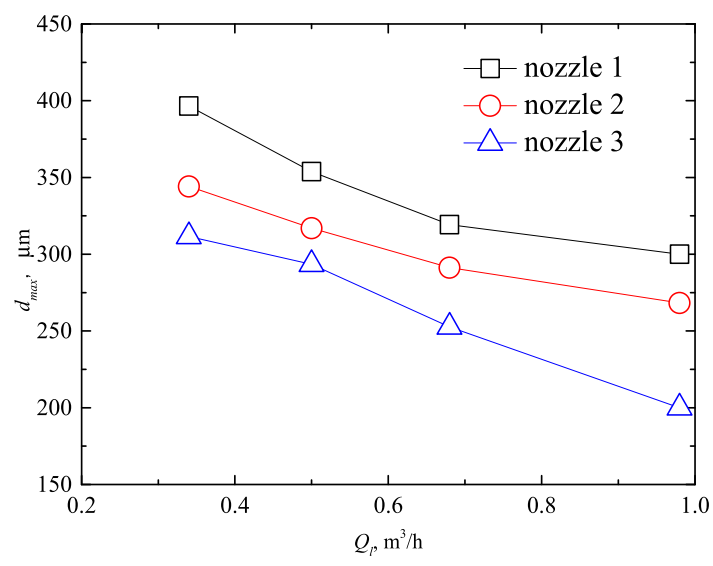

Figure 13. Effect of the inlet nozzle design on the maximum bubble diameter.

of the inlet nozzle. Owing to its sufficient degree of restriction, nozzle 3 induced the strongest swirl motion to the flow; thus, the bubble that could be separated by it was minimal. Combined with the effects of the gas and liquid flow rates on the maximum bubble diameter, the results indicate that the behavior of large bubbles depended on the swirl intensity. Increasing the gas and liquid flow rates, as well as the degree of restriction of the inlet nozzle, increased the swirl intensity, reducing the maximum bubble diameter.

4.2. Void Fraction Distribution. 4.2.1. Effects of Gas and Liquid Flow Rates on Bubble Migration. The rules governing the bubble migration in the swirling flow were investigated via ERT measurements. To confirm the reliability of ERT, a comparison between gas-core photographs and the cross-sectional void fraction distribution measured via ERT at an inlet gas flow rate of $55 \mathrm{~m}^{3} / \mathrm{h}$ was conducted (Figure 14).

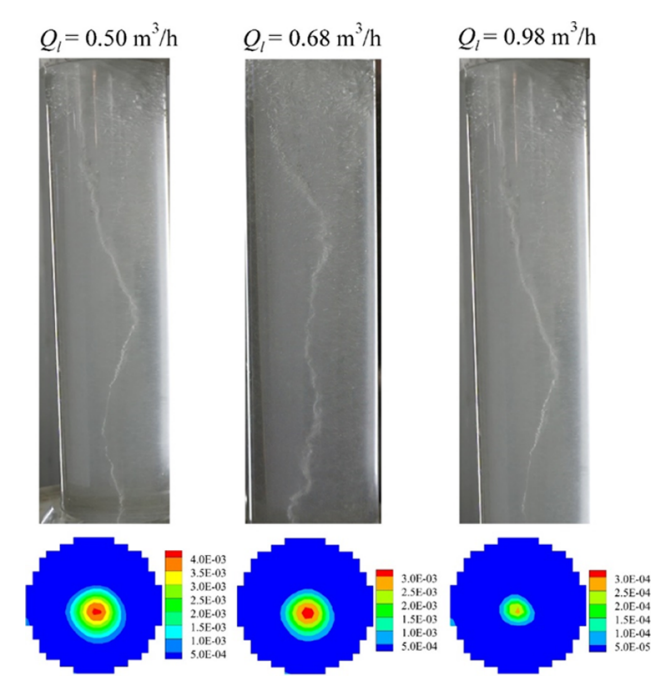

Figure 14. Gas-core photographs and the cross-sectional void fraction distribution measured using ERT at an inlet gas flow rate of $55 \mathrm{~m}^{3} / \mathrm{h}$.

Here, the red and blue colors represent high and low local void fractions, respectively. In these cases, nozzle 1 was used, and the liquid level was maintained at $0.70 \mathrm{~m}$. The gas core measured via ERT was slightly larger than that captured in the photograph. This was because the pixel resolution of the ERT electrode was slightly higher than that of the interface between the gas core and the liquid. However, the refraction of the plexiglass can visually narrow the gas core to a certain extent. Hence, overall, the cross-sectional void fraction distribution agrees well with the core distribution in the photograph, indicating that the phase distributions measured via ERT are reliable.

Moreover, the effect of the liquid flow rate on the void fraction was examined using Figure 14. Interestingly, the gas core narrowed with the augmentation of the liquid flow rate when the gas flow rate was fixed. To investigate this phenomenon further, the local void fraction distributions along the horizontal centerline for different liquid flow rates were plotted, as shown in Figure 15. The local void fraction

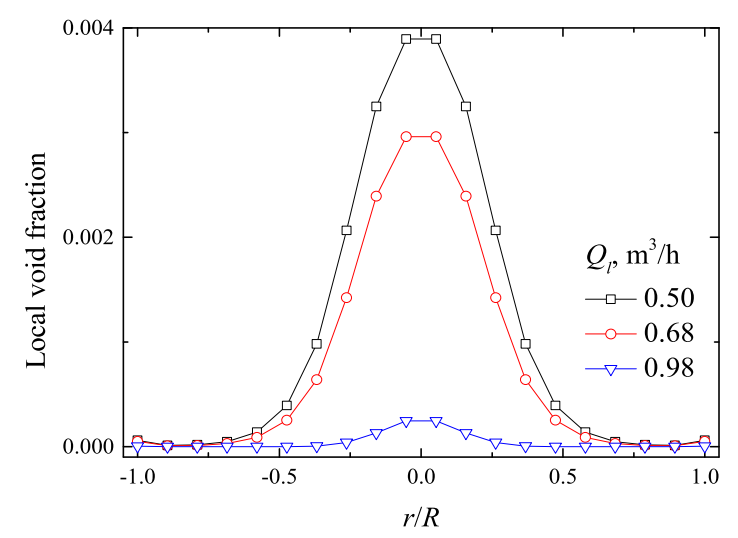

Figure 15. Local void fraction distribution along the horizontal centerline at an inlet gas flow rate of $55 \mathrm{~m}^{3} / \mathrm{h}$.

distribution at a liquid flow rate of $0.68 \mathrm{~m}^{3} / \mathrm{h}$ was slightly narrower than that at a liquid flow rate of $0.50 \mathrm{~m}^{3} / \mathrm{h}$, but both were significantly larger than that at a liquid flow rate of 0.98 $\mathrm{m}^{3} / \mathrm{h}$. There was almost no gas left in the ERT sampling section at the highest liquid flow rate. These phenomena can be physically explained by the axial velocity profile for the lower part of the GLCC. In the lower part of the GLCC, there was a flow-reversal region near the pipe center, which was determined using the axial velocities across the GLCC diameter measured via laser Doppler velocimetry (LDV) at 24 different axial locations. ${ }^{31}$ After the bubbles migrated to upward flow regions, they returned to the free interface. This explanation can be verified using the void fraction distributions of the higher and lower ERT sampling sections (Figure 16. See also video "flow reversal"). Figure 17 depicts the corresponding local void fraction distribution along the horizontal centerline. The average void fractions $\left(\alpha_{\mathrm{h}}\right.$ and $\left.\alpha_{1}\right)$ are also marked in the figure. In this case, nozzle 3 (with a high swirl intensity) was used to highlight the differences in the void fraction distributions between the higher and lower ERT sampling sections. The gas-core region and average gas holdup were broader in the higher ERT sampling section than in the lower ERT sampling section, indicating the existence of a flowreversal region in the gas core. Higher liquid flow rates contributed to a higher tangential velocity, which significantly increased the speed of the bubble migration in the radial direction. Before arriving in the measurement section, most of the bubbles reached the flow-reversal regions and moved back to the free interface. Therefore, the gas-core size decreased as the liquid flow rate increased.

Figure 18 displays the cross-sectional void fraction distribution at different gas flow rates and a fixed liquid flow rate. In these cases, nozzle 1 was used, and the liquid level was 


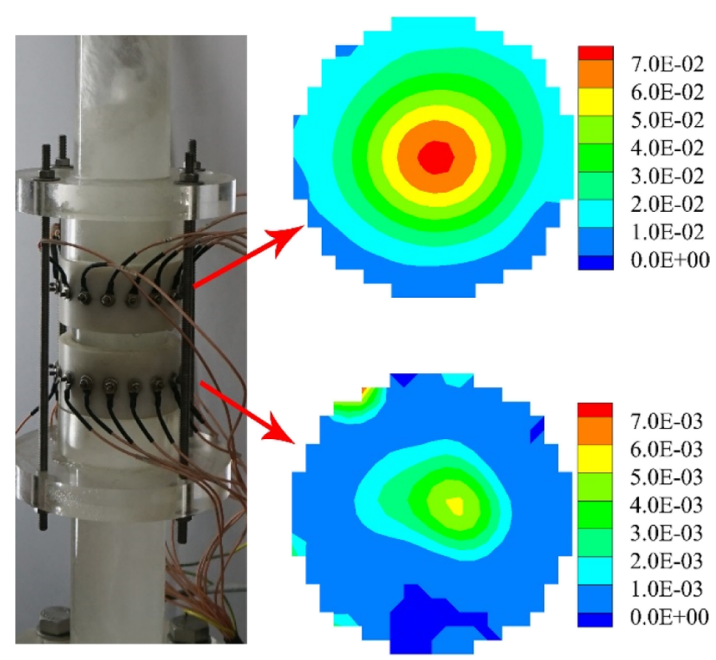

Figure 16. Cross-sectional void fraction distribution in the higher and lower ERT sampling sections.

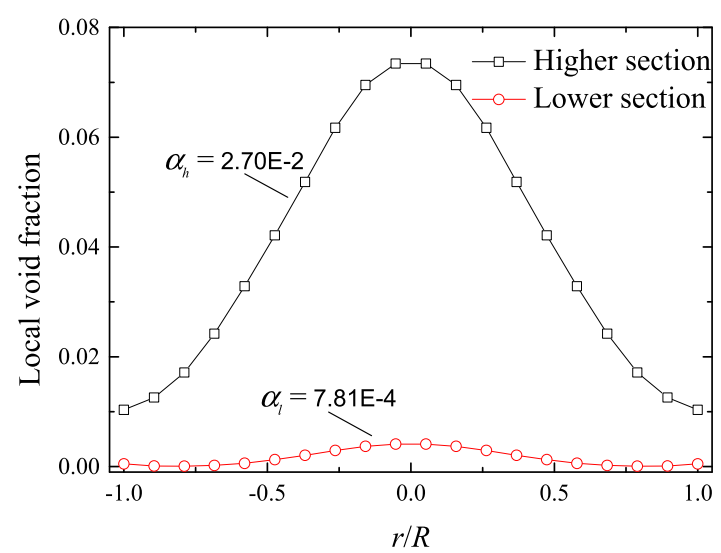

Figure 17. Local void fraction distribution along the horizontal centerline of the higher and lower ERT sampling sections.

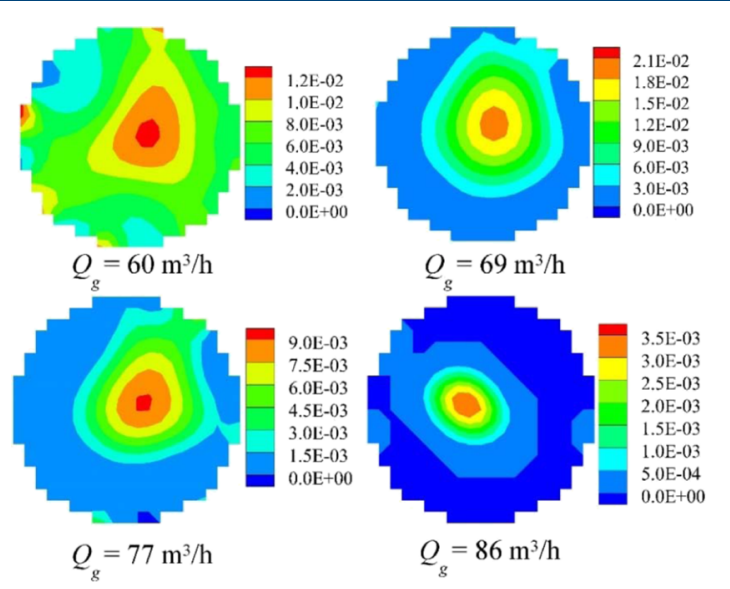

Figure 18. Measured cross-sectional void fraction distribution at an inlet liquid flow rate of $0.68 \mathrm{~m}^{3} / \mathrm{h}$.

maintained at $0.70 \mathrm{~m}$. Correspondingly, Figure 19 shows the radial distribution of the local void fraction. Better convergence of the gas core was observed when the gas flow rate increased from 60 to $69 \mathrm{~m}^{3} / \mathrm{h}$. However, the average gas holdup appeared not to have changed significantly $\left(6.73 \times 10^{-3}\right.$ and $5.19 \times 10^{-3}$, respectively), indicating that the degree of flow reversal in the gas core had little effect. Subsequently, the gas-

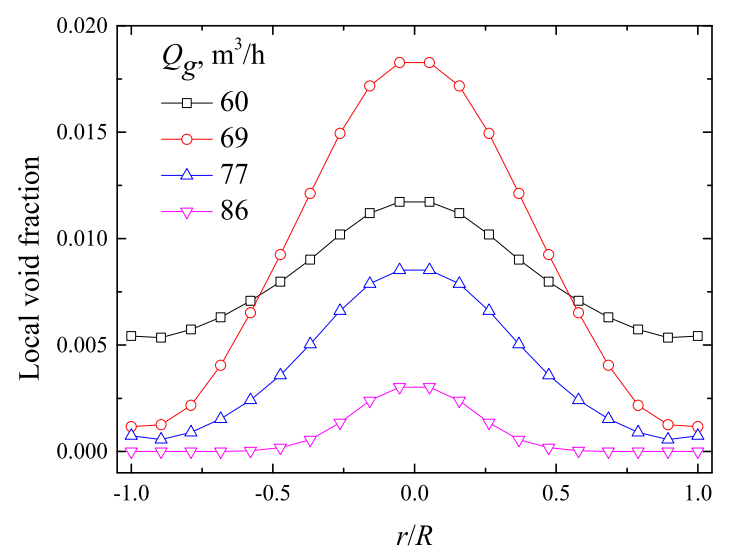

Figure 19. Local void fraction distribution along the horizontal centerline at an inlet liquid flow rate of $0.68 \mathrm{~m}^{3} / \mathrm{h}$.

core size and the void fraction magnitude decreased gradually as the gas flow rate increased from 69 to $86 \mathrm{~m}^{3} / \mathrm{h}$. The higher gas flow rates induced denser bubbles near the free interface (discussed previously), which theoretically increased the gascore size. However, owing to the reduction in the sectional area occupied by the liquid phase and the effect of the drag force, the swirl intensity increased as the gas flow rate increased. This promoted the convergence of bubbles within a shorter distance. The enhancement effect of the swirl intensity exceeded the effect of the increased bubble density; thus, the gas-core size decreased with an increase in the gas flow rate.

4.2.2. Effect of Liquid Level on Bubble Separation. The separation performance of a GLCC is strongly affected by the liquid level. In this study, the liquid level was controlled by adjusting the differential pressure between the two outlets. When the liquid level was approximately $0.31 \mathrm{~m}(0.18 \mathrm{~m}$ above the centerline of the liquid outlet), the flow patterns in the lower part of the GLCC were annular flow above the free interface and bubble flow below the free interface (Figure 20.
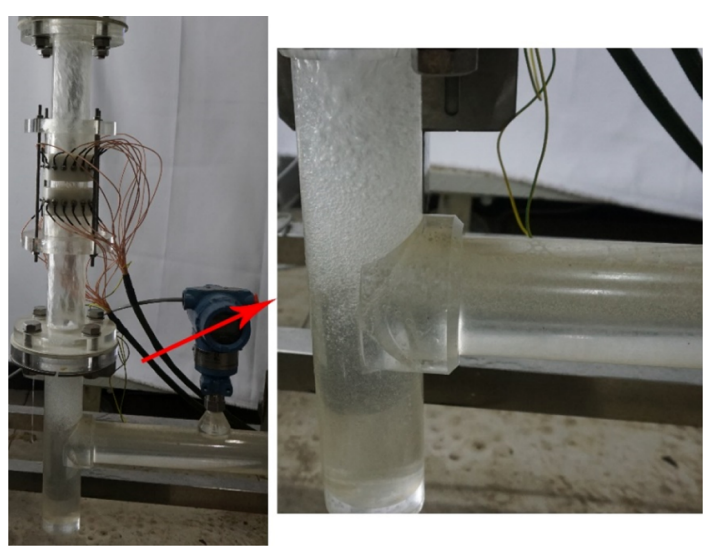

Figure 20. Flow pattern when the liquid level was $0.31 \mathrm{~m}$.

See also video "annular flow"). Owing to the severe attenuation of the swirl intensity, the bubbles dispersed rather than converged. The corresponding gas volume fraction at the liquid outlet was high. When the liquid level increased to 0.64 $\mathrm{m}$, the gas core started to appear, as shown in Figure 21. However, without a sufficient distance for bubbles to migrate, the void fraction at the ERT sampling section remained high. With a further increase in the liquid level $(0.82 \mathrm{~m})$, the gas core became smaller, and almost all bubbles accumulated near 


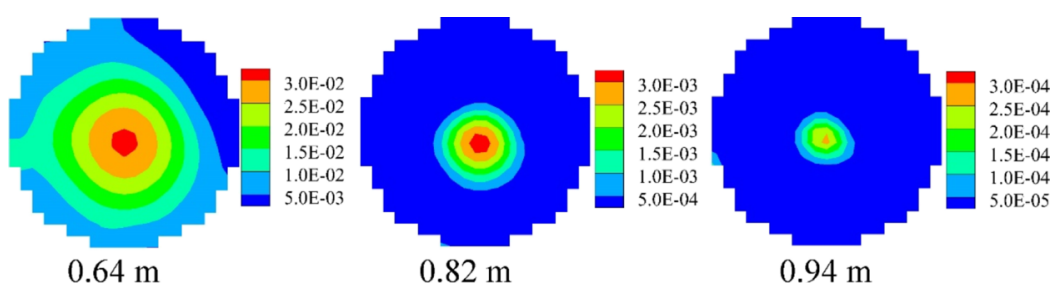

Figure 21. Effect of the liquid level on the measured cross-sectional void fraction distribution.

the pipe center. In this situation, the gas volume fraction at the liquid outlet reached almost its minimum value. When the liquid level increased to $0.94 \mathrm{~m}(0.03 \mathrm{~m}$ above the inlet centerline), few bubbles remained at the ERT sampling section (see Figures 21 and 22). However, in this case, the flow was

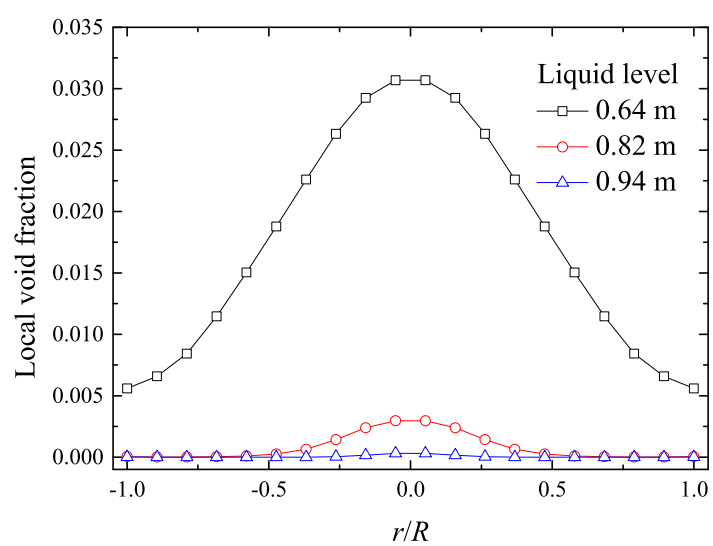

Figure 22. Local void fraction distribution along the horizontal centerline for different liquid levels.

turbulent at the inlet, and a large amount of liquid was carried upward with the gas stream (Figure 23. See also video "turbulent flow"). In conclusion, when the liquid level was near $0.82 \mathrm{~m}$, the optimal gas-liquid separation performance was achieved.

4.2.3. Effect of Inlet Nozzle Design on Void Fraction Distribution. Figure 24 shows the effect of the inlet nozzle design on the measured cross-sectional void fraction distribution. In these cases, the liquid level was maintained at $0.70 \mathrm{~m}$. The gas core was wide when nozzle 1 was employed. The swirl intensity of nozzle 1 was low owing to its insufficient restriction. Although a gas core formed, many of the bubbles were still dispersed around the gas core. The swirl intensity was strengthened by using nozzle 2 . Bubbles migrated more rapidly to the pipe center, and the amount of dispersed bubbles decreased. Hence, the gas core became narrower and more stable. However, the restriction was too severe with nozzle 3, which increased the turbulence at the inlet. The bubbles formed near the free interface were dense and small under the high turbulence. Moreover, the strong swirling flow induced a large shear force on the bubbles; thus, the bubbles in the GLCC were more likely to break up. The decrease in the bubble size likely counteracted the enhancement due to the centrifugal effect. Additionally, the downward velocities of the gas and liquid both increased as the degree of nozzle restriction increased, owing to the downward-inclined design. This was verified by LDV measurements in the lower part of the GLCC. $^{32}$ In situations with a high swirl intensity, bubbles in the gas core are carried toward the liquid outlet rather than toward the free interface.

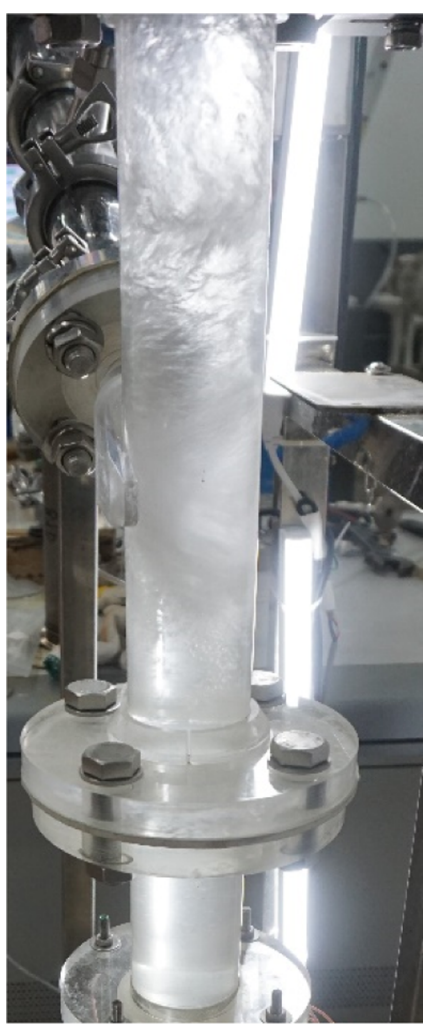

Figure 23. Turbulent flow when the liquid level was $0.94 \mathrm{~m}$.

Further discussion is motivated by the local void fraction along the horizontal centerline of the ERT sampling section depicted in Figure 25. The average void fractions $\left(\alpha_{1}, \alpha_{2}\right.$, and $\left.\alpha_{3}\right)$ are marked in the figure. In contrast with nozzles 1 and 2, a wider gas core and a higher cross-sectional void fraction were induced by nozzle 3 . Compared with nozzle 1 , bubbles were more concentrated in the pipe center for nozzle 2 , owing to the higher swirl intensity. The average void fractions for nozzles 1 and 2 were essentially identical but were significantly lower than that for nozzle 3. This confirms the foregoing discussion.

4.3. Separation Mechanism of Bubbles. Many structural and operational parameters can affect the separation performance of a GLCC. However, the gas-liquid separation is directly related to the velocity and pressure distribution in the swirling flow field. The velocity and pressure distribution were obtained for studying the separation mechanism of bubbles by numerical simulation. Figure 26 shows the radial distributions of the axial and tangential velocities in three different sections: $0.40,0.60$, and $0.80 \mathrm{~m}$ above the bottom of the GLCC. The tangential velocity can be approximately regarded as the combination of free and forced vortices, which is similar to the Rankine vortex. The pressure distribution profile also behaved as a Rankine vortex; that is, the pressure decreased with the decreasing radius, as shown in Figure 27. 

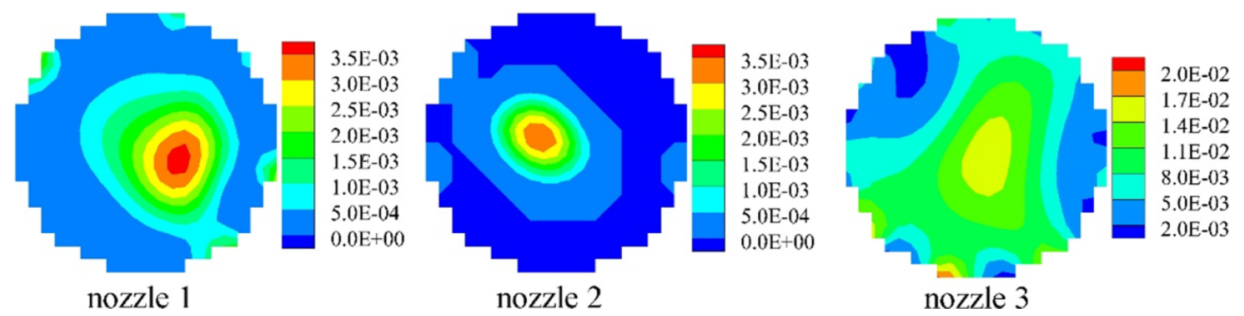

Figure 24. Effect of the inlet nozzle design on the measured cross-sectional void fraction distribution.

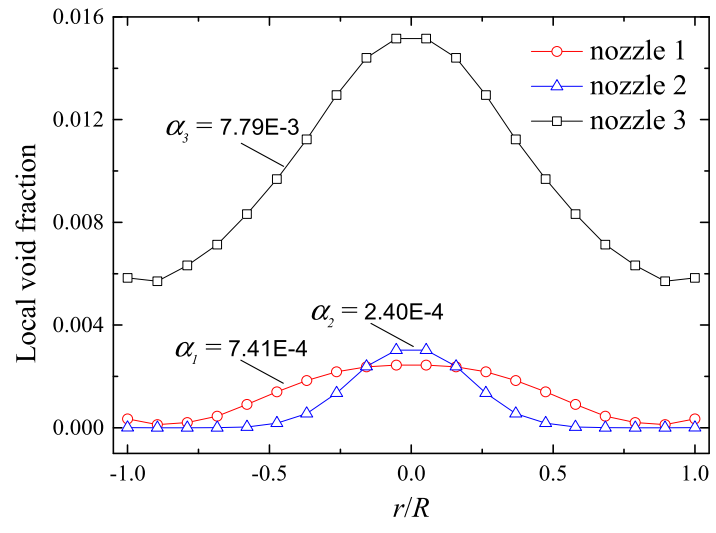

Figure 25. Local void fraction distribution along the horizontal centerline for different inlet nozzles.

The pressure gradient resulted in light bubbles being translated more quickly toward the pipe center than the heavy liquid phase, which was the main reason for gas-liquid separation. According to the characteristics of vortex motion, a higher tangential velocity can lead to a greater pressure gradient. As shown in Figure 26, the tangential velocity was quickly attenuated along the axis owing to the decay of the swirl intensity. Correspondingly, the pressure gradient decreased sharply as the axial height decreased, as shown in Figure 27. Thus, the gas-liquid separation effect was greatest near the inlet. Figure 28 depicts the variation of the pressure along the central axis. Generally, the pressure far from the inlet was higher than that near the inlet. Thus, a flow-reversal

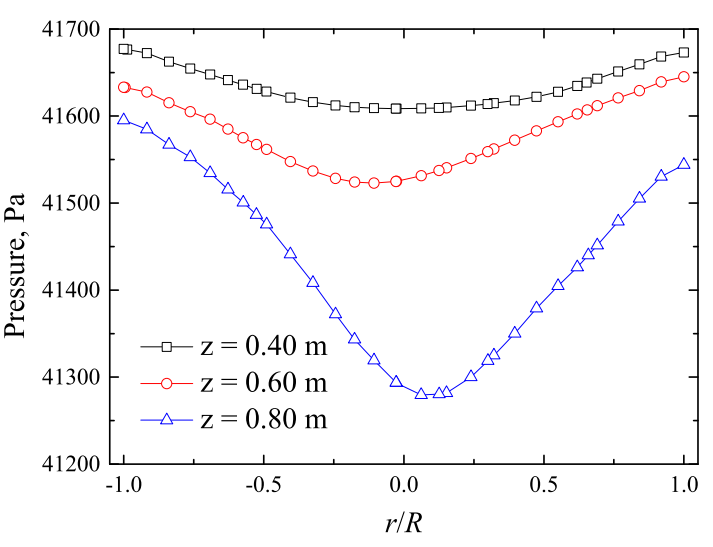

Figure 27. Simulated pressure distributions along the horizontal centerline in three different sections $(0.40,0.60$, and $0.80 \mathrm{~m})$ above the bottom of the GLCC.

phenomenon occurred in the region of the pipe center, as indicated by the axial velocity distribution in Figure 26.

Generally, the tangential velocity pushed the bubbles toward the center, forming the gas core, which promoted the separation of the bubbles from the liquid phase. The axial velocity dragged the dispersed bubbles toward the liquid outlet, which had a negative effect on the gas-liquid separation. If the bubbles migrated to the gas core before reaching the liquid outlet, they could flow back to the free interface and disengage. Conversely, the bubbles that flowed out with the liquid stream caused GCU. Therefore, the balance between the tangential velocity and the axial velocity

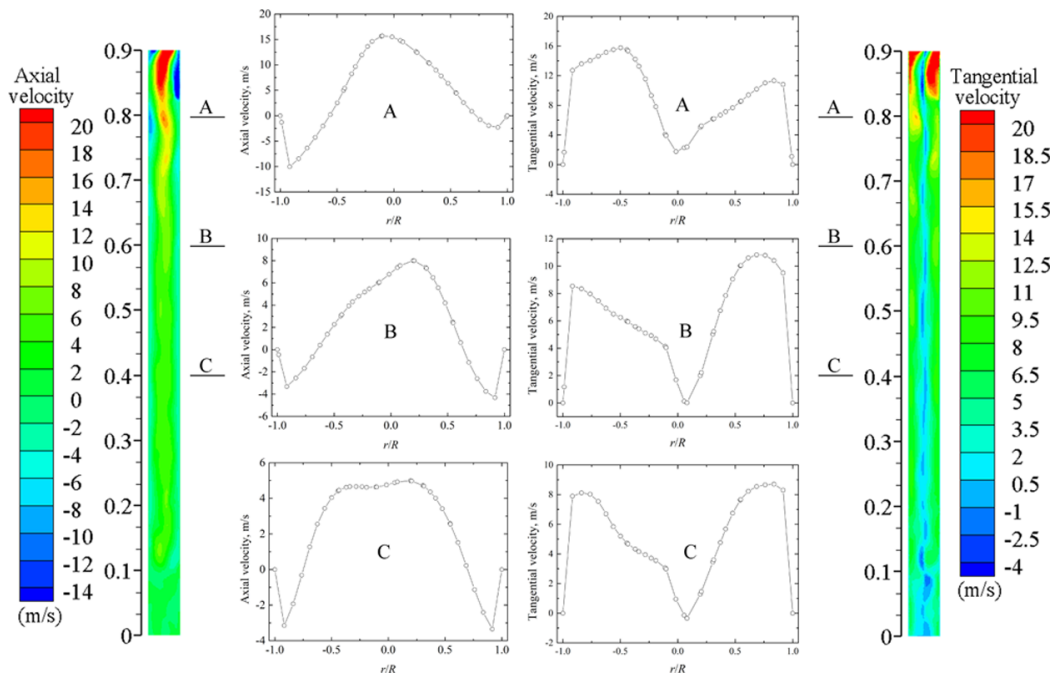

Figure 26. Simulated radial distributions of the axial and tangential velocities in three different sections $(0.40,0.60$, and $0.80 \mathrm{~m})$ above the bottom of the GLCC. 


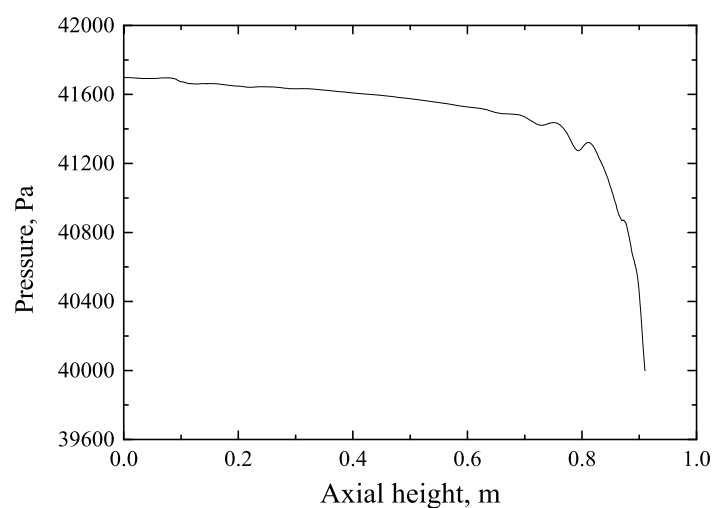

Figure 28. Simulated pressure variation along the central axis.

determined the gas-liquid separation efficiency. This conclusion can be verified by the gas volume fraction at the liquid outlet $\left(F_{\mathrm{gl}}\right)$ for different inlet nozzles, which is shown in Figure 29 . For nozzle 1 , the tangential velocity was especially low at

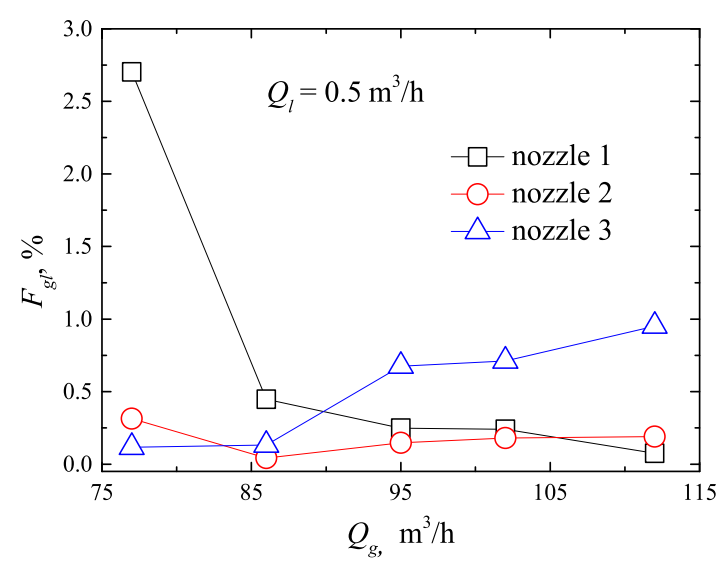

Figure 29. Measured gas volume fraction at the liquid outlet for different inlet nozzles.

low gas flow rate. The tangential velocity increased with an increase in the gas flow rate. Therefore, for nozzle 1 , the gas volume fraction at the liquid outlet decreased from 2.7 to $0.07 \%$ when the gas flow rate increased from 77 to $112 \mathrm{~m}^{3} / \mathrm{h}$. For nozzle 3 with severe restriction, when the gas flow rate increased to $95 \mathrm{~m}^{3} / \mathrm{h}$, the downward axial velocity became too high, and the gas volume fraction at the liquid outlet also became large. Nozzle 2 had a moderate restriction, and there existed a good balance between the tangential velocity and the axial velocity; thus, the gas volume fraction at the liquid outlet was always low at most cases.

4.4. Mechanism Model. According to the foregoing discussion, a bubble migration model was developed to predict the bubble trajectory in the lower part of the GLCC. As shown in Figure 30, the bubbles were assumed to be released from the wall at the bottom of the free interface. Once a bubble arrives at the axis of the GLCC, the bubble is assumed to be captured.

The swirl intensity can be characterized by the ratio of the tangential momentum flux to the total momentum flux, as follows

$$
\Omega=\frac{2 \pi \rho \int_{0}^{\mathrm{P}} v_{\mathrm{sl}} v_{\mathrm{ct}}(r) r \mathrm{~d} r}{\pi \rho R^{2} v_{\mathrm{sl}}^{2}}
$$

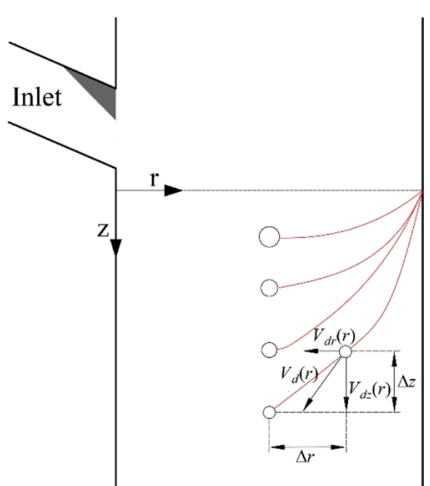

Figure 30. Schematic of the bubble migration model for the GLCC.

In this work, the prediction of the swirl intensity was based on the relationship developed by Chang and Dhir ${ }^{33}$

$$
\Omega=1.48\left(\frac{M_{\mathrm{t}}}{M_{\mathrm{T}}}\right)^{0.93} \exp \left[-0.113\left(\frac{M_{\mathrm{t}}}{M_{\mathrm{T}}}\right)^{0.35}\left(\frac{z}{D}\right)^{0.7}\right],
$$$$
\text { for } z / D \geq 2
$$

where $D$ represents the diameter of a GLCC. Here, $M_{t} / M_{T}$ represents the ratio of the momentum flux at the inlet to the total momentum flux, which can be expressed as the ratio of the liquid tangential velocity at the inlet to that in the GLCC body.

The experimental results of Chang and Dhir ${ }^{33}$ indicate that for axial distances very close to the inlet $(z / D<2)$, the swirl intensity decays far more slowly. Consequently, the swirl intensity can be regarded as a constant in this region.

For eq 19, the parameters in this study can be calculated as follows

$$
\begin{aligned}
& \frac{M_{\mathrm{t}}}{M_{\mathrm{T}}}=\frac{v_{\mathrm{il}}}{v_{\mathrm{sl}}} \\
& v_{\mathrm{sl}}=\frac{Q_{1}}{\pi D^{2} / 4} \\
& v_{\mathrm{il}}=\frac{Q_{\mathrm{g}}+Q_{1}}{\pi D^{2} / 4}\left(\frac{Q_{\mathrm{g}}}{Q_{1}}\right)^{-0.3}
\end{aligned}
$$

Because the zone of the free vortex is short (Figure 24), a linear radial distribution of the tangential velocity can be applied

$$
v_{\mathrm{ct}}(r)=\frac{r}{R} v_{\mathrm{ctmax}}
$$

where $v_{\text {ctmax }}$ represents the maximum tangential velocity in the radial direction.

According to eqs 18 and 23, the local swirl intensity can be calculated as

$$
\Omega=\frac{2}{3} \frac{v_{\mathrm{ctmax}}}{v_{\mathrm{sl}}}
$$

Therefore, the obtained tangential velocity distribution is in the following form

$$
v_{\mathrm{ct}}(r)=\frac{3 r v_{\mathrm{sl}} \Omega}{D}
$$




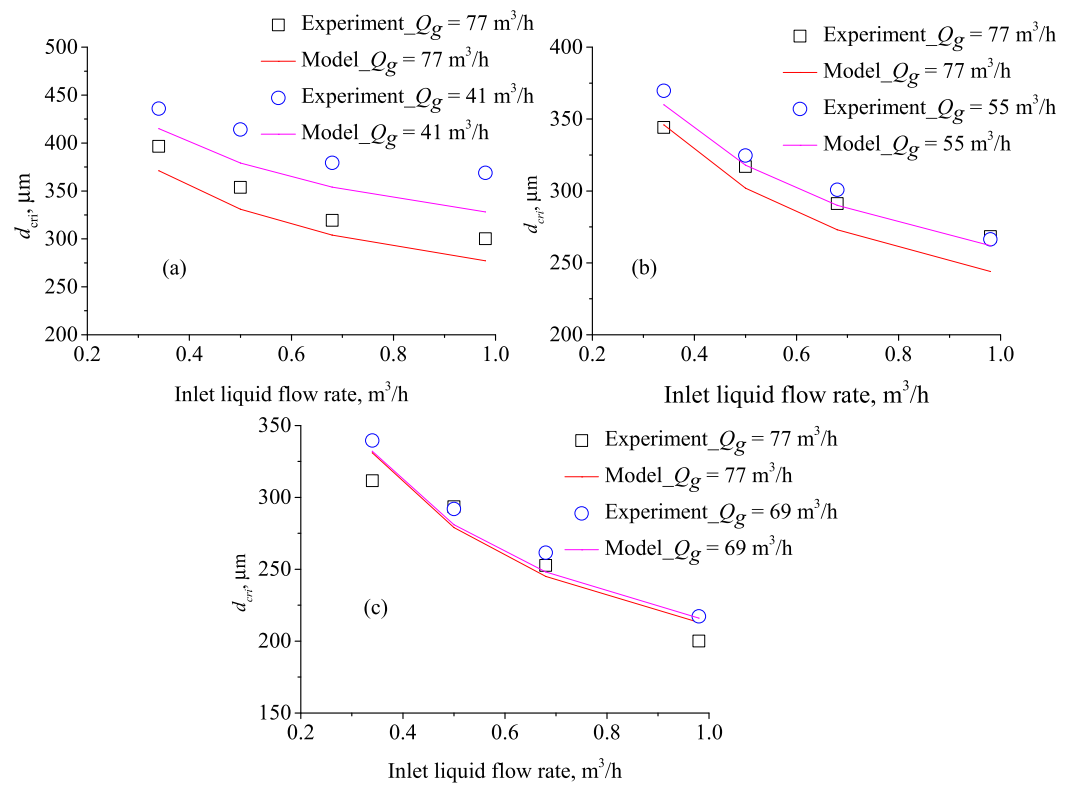

Figure 31. Comparison between the predicted values and experimental data for the critical diameter of bubbles: (a) nozzle 1, (b) nozzle 2, (c) nozzle 3.

In this work, the velocity of the bubble was further determined by the force analysis of the bubble. The drag force of the bubble is calculated as

$$
F_{\mathrm{D}}=\frac{1}{2} C_{\mathrm{D}} \rho_{1} v_{\mathrm{d}}{ }^{2} \frac{\pi}{4} d^{2}
$$

where $C_{\mathrm{D}}$ is the drag coefficient, $\rho_{1}$ represents the liquid-phase density, $d$ represents the bubble diameter, and $v_{\mathrm{d}}$ represents the resultant slip velocity of the bubble.

The resultant slip velocity of the bubble is expressed as

$$
v_{\mathrm{d}}(r)=\sqrt{v_{\mathrm{dr}}^{2}(r)+v_{\mathrm{dz}}^{2}(r)}
$$

where $v_{\mathrm{dr}}$ represents the radial slip velocity and $v_{\mathrm{dz}}$ represents the axial slip velocity.

The drag coefficient is calculated using the correlation developed by Magnaudet ${ }^{34}$

$$
C_{\mathrm{D}}=\frac{16}{\operatorname{Re}}\left\{1+\left[\frac{8}{\operatorname{Re}}+\frac{1}{2}\left(1+3.315 \operatorname{Re}^{-1 / 2}\right)\right]^{-1}\right\}
$$

The Reynolds number is given as

$$
R e=\frac{\rho_{1} v_{\mathrm{d}} d}{\mu_{1}}
$$

where $\mu_{1}$ is the liquid viscosity coefficient.

The centrifugal force plays a vital role in gas-liquid separation. The direction of the centrifugal force is from the axis to the wall. This force is expressed as follows

$$
F_{\mathrm{C}}=\frac{\pi}{6} d^{3} \rho_{\mathrm{g}} \frac{v_{\mathrm{ct}}^{2}}{r}
$$

where $\rho_{\mathrm{g}}$ represents the gas-phase density.

The force induced by the pressure gradient yields

$$
F_{\mathrm{rc}}=\frac{\pi}{6} d^{3} \rho_{1} \frac{v_{\mathrm{ct}}^{2}}{r}
$$

The resultant force of gravity and buoyancy in the axial direction of the bubbles is expressed as

$$
F_{\mathrm{cz}}(r)=\frac{\pi}{6} d^{3}\left(\rho_{1}-\rho_{\mathrm{g}}\right) g
$$

According to the force balance in the radial direction, the radial slip velocity for a bubble can be obtained as follows

$$
v_{\mathrm{dr}}(r)=\frac{12 \Omega}{D^{2}}\left(\frac{\rho_{1}-\rho_{\mathrm{g}}}{\rho_{\mathrm{g}}}\right) \frac{d}{C_{\mathrm{D}}} \frac{v_{\mathrm{sl}}{ }^{2}}{v_{\mathrm{d}} r}
$$

Similarly, the axial slip velocity for a bubble can be calculated

$$
v_{\mathrm{dz}}(r)=\frac{4}{3}\left(\frac{\rho_{1}-\rho_{\mathrm{g}}}{\rho_{\mathrm{g}}}\right) g \frac{d}{C_{\mathrm{D}}} \frac{1}{v_{\mathrm{d}}}
$$

Solving for the bubble velocity yields

$$
v_{\mathrm{d}}(r)=\sqrt{\frac{4}{3}\left(\frac{\rho_{1}-\rho_{\mathrm{g}}}{\rho_{\mathrm{g}}}\right) \frac{d}{C_{\mathrm{D}}}}\left[g^{2}+\left(\frac{9 r v_{\mathrm{sl}}^{2} \Omega}{D^{2}}\right)^{2}\right]^{1 / 4}
$$

Once $v_{\mathrm{d}}$ is determined, the radial and axial slip velocities can be calculated using eqs 33 and 34, respectively.

During the time interval $\Delta t$, the bubble migrates $\Delta r=$ $v_{\mathrm{dr}}(r) \Delta t$ in the radial direction and $\Delta z_{\mathrm{d}}=\left[v_{\mathrm{sl}}-v_{\mathrm{dz}}(r)\right] \Delta t$ in the axial direction. The governing equation for the axial migration displacement can be determined by combining these two equations. The entire trajectory of the bubble can be mapped by integrating the governing equation, that is

$$
\Delta z_{\mathrm{d}}=\int \frac{v_{\mathrm{sl}}-v_{\mathrm{dz}}(r)}{v_{\mathrm{dr}}(r)} \mathrm{d} r
$$

The trajectory of the bubble can be employed to determine whether the bubble migrates radially to the pipe center and is separated from the liquid phase. The critical diameter of bubbles that can reach the gas core is defined as $d_{\text {cri }}$. Bubbles smaller than $d_{\text {cri }}$ are carried away in the liquid stream. 
The bubble size measured by the Malvern RTsizer was used to validate the mechanism model developed in this study. Figure 31 presents a comparison between the predicted and experimental results for different inlet nozzles. As shown, for the different inlet nozzles and inlet conditions, the predicted critical bubble diameters agreed well with the experimental data. $87.5 \%$ of the data have an average absolute error less than $8 \%$, as seen from the error analysis in Figure 32. This indicates that the bubble trajectory can be accurately predicted by the developed model.

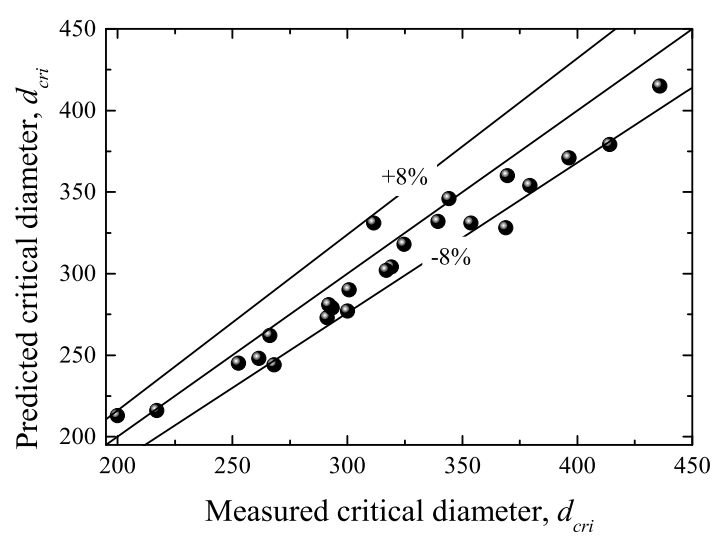

Figure 32. Error analysis of the developed mechanism model.

\section{CONCLUSIONS}

Experiments and numerical simulations were conducted to investigate the bubble behavior in the lower part of the GLCC, particularly the breakup, coalescence, and migration regularity of bubbles. The following conclusions were drawn.

The bubble size distributions measured using a Malvern RTsizer indicated that small and medium-sized bubbles tended to initially break up and subsequently coalesce as the gas flow rate increased, whereas they always tended to break up as the liquid flow rate increased. Increasing the gas and liquid flow rates, as well as the degree of restriction of the inlet nozzle, increased the swirl intensity, reducing the maximum bubble diameter.

The rules affecting the bubble migration in the swirling flow were investigated via ERT measurements. Owing to the flow reversal in the core, the gas core narrowed with an increase in the liquid flow rate. As the gas flow rate increased, the enhancement effect from the swirl intensity exceeded the effect of the increased bubble density; thus, the gas core became smaller. When the liquid level was near $0.82 \mathrm{~m}$, the optimal gas-liquid separation performance was achieved. Both insufficient and excessive nozzle restrictions had negative effects on the bubble separation.

The DPM model coupled with the RSM turbulence model was used to simulate the swirling flow field characteristics. The separation mechanism of bubbles was analyzed according to the experimental observations and the simulated flow field distribution. Finally, a mechanism model was developed for predicting the critical diameter of bubbles that could be separated in a GLCC, according to analysis of the bubble migration. The developed model gave a reasonable performance, with $87.5 \%$ of the data having an average absolute error less than $8 \%$.

\section{ASSOCIATED CONTENT}

\section{Supporting Information}

The Supporting Information is available free of charge at https://pubs.acs.org/doi/10.1021/acs.iecr.9b05332.

Bubble coalescence (MP4)

Flow reversal (MP4)

Annular flow (MP4)

Turbulent flow (MP4)

\section{AUTHOR INFORMATION}

\section{Corresponding Author}

Li Zou - School of Naval Architecture, Dalian University of

Technology, Dalian 116024, China; Email: zoulidut@

163.com

\section{Authors}

Lele Yang - School of Marine Engineering and Technology, Sun Yat-sen University, Guangzhou 510275, China; Southern Marine Science and Engineering Guangdong Laboratory (Zhuhai), Zhuhai 519000, China

Yong Ma - School of Marine Engineering and Technology, Sun Yat-sen University, Guangzhou 510275, China; Southern Marine Science and Engineering Guangdong Laboratory (Zhuhai), Zhuhai 519000, China; @orcid.org/0000-00016663-3394

Jing Wang - School of Marine Engineering and Technology, Sun Yat-sen University, Guangzhou 510275, China

Jingyu Xu - Institute of Mechanics, Chinese Academy of Sciences, Beijing 100190, China

Complete contact information is available at:

https://pubs.acs.org/10.1021/acs.iecr.9b05332

\section{Notes}

The authors declare no competing financial interest.

\section{ACKNOWLEDGMENTS}

This work was financially supported by the China Postdoctoral Science Foundation (no. 2019M663242), the Fundamental Research Funds for the Central Universities (no. 19lgpy86), and the Dedicated Fund for Promoting High-Quality Economic Development in Guangdong Province (Marine Economic Development Project) (no. GDOE2019A43).

\section{REFERENCES}

(1) Hreiz, R.; Gentric, C.; Midoux, N. Numerical investigation of swirling flow in cylindrical cyclones. Chem. Eng. Res. Des. 2011, 89, $2521-2539$

(2) Hreiz, R.; Gentric, C.; Midoux, N.; Lainé, R.; Fünfschilling, D. Hydrodynamics and velocity measurements in gas-liquid swirling flows in cylindrical cyclones. Chem. Eng. Res. Des. 2014, 92, 22312246.

(3) Huang, L.; Deng, S.; Chen, Z.; Guan, J.; Chen, M. Numerical Analysis of a Novel Gas-Liquid Pre-Separation Cyclone. Sep. Purif. Technol. 2018, 194, 470-479.

(4) Hsiao, T.-C.; Huang, S.-H.; Hsu, C.-W.; Chen, C.-C.; Chang, P.$\mathrm{K}$. Effects of the geometric configuration on cyclone performance. $J$. Aerosol Sci. 2015, 86, 1-12.

(5) Kha, H. M.; Phuong, N. N.; Nam, N. T. The effect of different geometrical configurations of the performances of Gas-Liquid Cylindrical Cyclone separators (GLCC). International Conference on System Science and Engineering (ICSSE), 2017; pp 646-651.

(6) Kouba, G. E.; Shoham, O.; Shirazi, S. Design and performance of gas-liquid cylindrical cyclone separators. Proceedings of the BHR Group 
7th International Meeting on Multiphase Flow, June 7-9, 1995; BHR Group: Cannes, France, 1995; pp 307-327.

(7) Van Sy, L. Influence of inlet angle on flow pattern and performance of gas-liquid cylindrical cyclone separator. Part. Sci. Technol. 2017, 35, 555-564.

(8) Carlos Berrio, J.; Pereyra, E.; Ratkovich, N. Computational fluid dynamics modeling of gas-liquid cylindrical cyclones, Geometrical Analysis. J. Energy Resour. Technol. 2018, 140, 092003-092014.

(9) Molina, R.; Wang, S.; Gomez, L. E.; Mohan, R. S.; Shoham, O.; Kouba, G. Wet gas separation in gas-liquid cylindrical cyclone (GLCC) separator. Structures, Safety and Reliability; Petroleum Technology Symposium; ASME: San Diego, California, USA, 2007; Vol 2, pp 731-746.

(10) Kouba, G. E.; Shoham, O. A review of gas-liquid cylindrical cyclone (GLCC) technology. Production Separation Systems International Conference: Aberdeen, England, 1996; pp 23-24.

(11) Cornejo Caceres, J. S.; Prieto, N.; Gonzalez, G.; ChavesGuerrero, A. Numerical Simulation of a Natural Gas Cylindrical Cyclone Separator Using Computational Fluid Dynamics. Ind. Engi. Chem. Res. 2019, 58, 14323-14332.

(12) Moncayo, J. A.; Dabirian, R.; Mohan, R. S.; Shoham, O.; Kouba, G. Foam break-up under swirling flow in inlet cyclone and GLCC. J. Pet. Sci. Eng. 2018, 165, 234-242.

(13) Kolla, S. S.; Mohan, R. S.; Shoham, O. Experimental investigation of liquid carry-over in GLCC(C) separators for 3-phase flow. Fluids Engineering; ASME: Phoenix, Arizona, USA, 2016; Vol. 7, V007T09A006.

(14) Wang, S.; Gomez, L. E.; Mohan, R. S.; Shoham, O.; Kouba, G. E. Gas-Liquid Cylindrical Cyclone (GLCC) Compact Separators For Wet Gas Applications. J. Energy Resour. Technol. 2003, 125, 43-50.

(15) Yue, T.; Chen, J.; Song, J.; Chen, X.; Wang, Y.; Jia, Z.; Xu, R. Experimental and numerical study of upper swirling liquid film (USLF) among gas-liquid cylindrical cyclones (GLCC). Chem. Eng. J. 2019, 358, 806-820.

(16) Chirinos, W. A.; Gomez, L. E.; Wang, S.; Mohan, R. S.; Shoham, O.; Kouba, G. E. Liquid Carry-Over in Gas/Liquid Cylindrical Cyclone Compact Separators. SPE J. 2000, 5, 259-267.

(17) Reyes-Gutiérrez, M. A.; Rojas-Solórzano, L. R.; Marín-Moreno, J. C.; Meléndez-Ramírez, A. J.; Colmenares, J. Eulerian-Eulerian Modeling of Disperse Two-Phase Flow in a Gas-Liquid Cylindrical Cyclone. J. Fluids Eng. 2005, 128, 832-837.

(18) Erdal, F. M.; Shirazi, S. A.; Mantilla, I.; Shoham, O. CFD Study of Bubble Carry-Under in Gas-Liquid Cylindrical Cyclone Separators. SPE Annual Technical Conference and Exhibition, 1998; Vol. 15, pp 217-222.

(19) Simmons, M. J. H.; Hanratty, T. J. Droplet size measurements in horizontal annular gas-liquid flow. Int. J. Multiphase Flow 2001, 27, $861-883$.

(20) Simmons, M. J. H.; Zaidi, S. H.; Azzopardi, B. J. Comparison of laser-based drop-size measurement techniques and their application to dispersed liquid-liquid pipe flow. Opt. Eng. 2000, 39, 505-509.

(21) Liu, S.; Yang, L.-l.; Zhang, D.; Xu, J.-y. Separation characteristics of the gas and liquid phases in a vane-type swirling flow field. Int. J. Multiphase Flow 2018, 107, 131-145.

(22) Wang, H.; Jia, J.; Yang, Y.; Buschle, B.; Lucquiaud, M. Quantification of gas distribution and void fraction in packed bubble column using electrical resistance tomography. IEEE Sens. J. 2018, 18, 8963-8970.

(23) Jia, J.; Wang, M.; Faraj, Y.; Wang, Q. Online conductivity calibration methods for EIT gas/oil in water flow measurement. Flow Meas. Instrum. 2015, 46, 213-217.

(24) Jia, J.; Wang, M.; Faraj, Y. Evaluation of eit systems and algorithms for handling full void fraction range in two-phase flow measurement. Meas. Sci. Technol. 2015, 26, 015305.

(25) Wang, M.; Dickin, F. J.; Mann, R. Electrical resistance tomographic sensing systems for industrial applications. Chem. Eng. Commun. 1999, 175, 49-70.
(26) Liu, S.; Zhang, D.; Yang, L.-1.; Xu, J.-y. Breakup and coalescence regularity of non-dilute oil drops in a vane-type swirling flow field. Chem. Eng. Res. Des. 2018, 129, 35-54.

(27) Morsi, S. A.; Alexander, A. J. An Investigation of Particle Trajectories in Two-Phase Flow Systems. J. Fluid Mech. 1972, 55, 193-208.

(28) O’Rourke, P. J. Collective Drop Effects on Vaporizing Liquid Sprays; Los Alamos National Laboratory: NM, USA, 1981.

(29) Escue, A.; Cui, J. Comparison of turbulence models in simulating swirling pipe flows. Appl. Math. Model 2010, 34, 28402849.

(30) Zhang, J.; Xu, J.-Y. Rheological behaviour of oil and water emulsions and their flow characterization in horizontal pipes. Can. J. Chem. Eng. 2016, 94, 324-331.

(31) Erdal, F. M.; Shirazi, S. A. Effect of Inlet Configuration on Flow Behavior in a Cylindrical Cyclone Separator. Engineering Technology Conference on Energy, Parts A and B, ASME: Houston, Texas, USA, 2002; pp 521-529.

(32) Hreiz, R.; Gentric, C.; Midoux, N.; Lainé, R.; Fünfschilling, D. Hydrodynamics and velocity measurements in gas-liquid swirling flows in cylindrical cyclones. Chem. Eng. Res. Des. 2014, 92, 22312246.

(33) Chang, F.; Dhir, V. K. Turbulent flow field in tangentially injected swirl flows in tubes. Int. J. Heat Fluid Flow 1994, 15, 346356.

(34) Magnaudet, J. The forces acting on bubbles and rigid particles. ASME Fluids Engineering Division Summer Meeting, FEDSM, 1997; Vol. 97, pp 22-26. 